\title{
TWISTED BORCHERDS PRODUCTS ON HILBERT MODULAR SURFACES AND THEIR CM VALUES
}

\author{
JAN HENDRIK BRUINIER AND TONGHAI YANG
}

\begin{abstract}
We construct a natural family of rational functions $\tilde{\Psi}_{m}$ on a Hilbert modular surface from the classical $j$-invariant and its Hecke translates. These functions are obtained by means of a multiplicative analogue of the Doi-Naganuma lifting and can be viewed as twisted Borcherds products. We then study when the value of $\tilde{\Psi}_{m}$ at a CM point associated to a non-biquadratic quartic CM field generates the 'CM class field' of the reflex field. For the real quadratic field $\mathbb{Q}(\sqrt{5})$, we factorize the norm of some of these CM values to $\mathbb{Q}(\sqrt{5})$ numerically.
\end{abstract}

\section{INTRODUCTION}

In his papers [Bo1] and [Bo2] Borcherds constructed a lifting from certain weakly holomorphic elliptic modular forms of weight $1-n / 2$ to meromorphic modular forms on the orthogonal group of a rational quadratic space of signature $(2, n)$. Here we consider a related construction in the particular case that $n=2$.

Let $p$ be a prime congruent to 1 modulo 4 and let $F=\mathbb{Q}(\sqrt{p})$. We write $\mathcal{O}_{F}$ for the ring of integers and $\partial_{F}$ for the different of $F$. Considering the lattice $L^{0}=\mathbb{Z}^{2} \oplus \mathcal{O}_{F}$ in the rational quadratic space $L^{0} \otimes_{\mathbb{Q}} \mathbb{Q}$ of signature $(2,2)$, one obtains from Borcherds' result a lifting from weakly holomorphic elliptic modular forms of weight zero for the group $\Gamma_{0}(p)$ with Nebentypus character $\epsilon_{p}=(\dot{\bar{p}})$ to meromorphic Hilbert modular forms for the Hilbert modular group $\Gamma=\mathrm{SL}_{2}\left(\mathcal{O}_{F}\right)$ [Br1, $\mathrm{BB}$. This lifting can be viewed as a multiplicative analogue of the Naganuma lift from holomorphic modular forms of weight $k$ for $\Gamma_{0}(p)$ with Nebentypus $\epsilon_{p}$ to holomorphic Hilbert modular forms of weight $k$ for $\Gamma$ [Na, Za1].

There is another lifting from holomorphic elliptic modular forms to Hilbert modular forms, namely the celebrated Doi-Naganuma lift which maps holomorphic modular forms of weight $k$ for $\mathrm{SL}_{2}(\mathbb{Z})$ to holomorphic Hilbert modular forms of weight $k$ for $\Gamma$ [DN]. It was pointed out by Zagier that this lifting should have a multiplicative analogue as well [a3]. Moreover, Zagier stated several properties of such a multiplicative lifting and suggested that a proof could probably be given following the argument of [Br1]. One purpose of the present paper is to work out a proof along these lines.

Let $\mathbb{H}$ be the upper complex half plane, and put $q=e(\tau)=e^{2 \pi i \tau}$ for $\tau \in \mathbb{H}$. Recall that a weakly holomorphic modular form for $\mathrm{SL}_{2}(\mathbb{Z})$ is a meromorphic modular form for $\mathrm{SL}_{2}(\mathbb{Z})$ which is holomorphic outside the cusp $\infty$. In particular, every weakly holomorphic modular

Date: November 1, 2018.

2000 Mathematics Subject Classification. 11G15, 11F41, 14K22.

T.Y. Yang is partially supported by NSF grants DMS-0302043, 0354353, and a NSA grant. 
form of weight zero for $\mathrm{SL}_{2}(\mathbb{Z})$ is a polynomial in the $j$-function (which we normalize such that $\left.j(\tau)=q^{-1}+744+O(q)\right)$. In Section [5] we shall prove the following theorem. See Theorem 5.2 for a more detailed statement.

Theorem 1.1. Let $f=\sum_{n \gg-\infty} c(n) q^{n} \in \mathbb{Z}[j]$ be a weakly holomorphic modular form of weight 0 for $\mathrm{SL}_{2}(\mathbb{Z})$ with integral Fourier coefficients. Then there exists a symmetric meromorphic Hilbert modular function $\Psi(z, f)$ for $\Gamma$ (of weight 0 , with trivial multiplier system, defined over $F$ ) such that:

(i) The divisor of $\Psi(z, f)$ is determined by the polar part of $f$ at the cusp $\infty$. It equals $\sum_{n>0} c(-n) \tilde{T}_{n}$, where $\tilde{T}_{n}$ denotes the twisted Hirzebruch-Zagier divisor of discriminant $n$ defined in Section 3.

(ii) The function $\Psi(z, f)$ has the Borcherds product expansion

$$
\Psi(z, f)=\prod_{\substack{\nu \in \partial_{F}^{-1} \\ \nu>0}} \prod_{b(p)}\left(1-e\left(\frac{b}{p}+\nu z_{1}+\nu^{\prime} z_{2}\right)\right)^{\epsilon_{p}(b) c\left(p \nu \nu^{\prime}\right)}
$$

which converges normally for all $z=\left(z_{1}, z_{2}\right) \in \mathbb{H}^{2}$ with $\Im\left(z_{1}\right) \Im\left(z_{2}\right)>N p$ outside the set of poles, where $N=\max \{n \in \mathbb{Z} ; c(-n) \neq 0\}$. In particular, $\Psi(z, C)=1$ for any constant $C$.

(iii) The lifting is multiplicative, i.e., if $f, g \in \mathbb{Z}[j]$, then $\Psi(f+g)=\Psi(f) \Psi(g)$.

We now briefly describe the idea of the proof. For a positive integer $m$, we define in Section 3 a certain "twisted Hirzebruch-Zagier divisor" $\tilde{T}_{m}$ on the Hilbert modular surface corresponding to $\Gamma$ (see also [Za2] pp. 166). In Section 4, following Br1], we construct an automorphic Green function $\tilde{\Phi}_{m p^{2}}(z, s)$ for $\tilde{T}_{m}$. We study its main properties and compute its Fourier expansion (Theorems 4.4 and 4.6). By means of an identity relating certain finite exponential sums to Kloosterman sums (Lemma 4.3), we find that the Fourier coefficients of $\tilde{\Phi}_{m p^{2}}(z, s)$ are closely related to the coefficients of non-holomorphic Poincaré series of weight zero for $\mathrm{SL}_{2}(\mathbb{Z})$ (see Section 2). Using the fact that any weakly holomorphic modular form of weight zero can be uniquely written as a linear combination of the non-holomorphic Poincaré series, Theorem 1.1 can be deduced. Here the main point is an identity expressing $\log |\Psi(z, f)|$ as a linear combination of the automorphic Green functions $\tilde{\Phi}_{m p^{2}}(z, 1)$, see Theorem 5.2 (iv).

An alternative proof could be given by interpreting the lifting as a regularized theta lifting for the dual pair $\mathrm{SL}_{2}(\mathbb{R}), \mathrm{O}(2,2)$ as in $[\mathrm{Bo} 2, \mathrm{Br} 2$, and by considering suitable "twists" of Siegel theta functions as kernel functions. It would actually be very interesting to describe such twisted Borcherds liftings in greater generality for $\mathrm{O}(2, n)$. However, it seems not quite clear what the right "twists" of Siegel theta functions should be in general. We have not pursued this approach in the present paper, because the proof using automorphic Green functions leads to the result in a direct way. Moreover, the construction of the Green functions should be of independent interest.

If $m$ is a positive integer, we write $\tilde{\Psi}_{m}$ for the symmetric Hilbert modular function of weight 0 which is the twisted Borcherds lift via Theorem [1.1] of the unique weakly 
holomorphic modular form

$$
J_{m}=q^{-m}+O(q) \in \mathbb{Z}[j]
$$

In this way we obtain a 'canonical' family of rational functions on the Hilbert modular surface associated to $\Gamma$. It seems natural to ask for their arithmetic properties. For instance, one can ask whether $\tilde{\Psi}_{1}$, the lifting of the $j$-function, has a nice moduli interpretation as an invariant of abelian surfaces with $\mathcal{O}_{F}$-multiplication and $\partial_{F}^{-1}$-polarization.

It is a well-known and beautiful fact that $j\left(\frac{D+\sqrt{-D}}{2}\right)$ generates the Hilbert class field of the imaginary quadratic field $\mathbb{Q}(\sqrt{-D})$. In Section $\left[\right.$, we study when the value of $\tilde{\Psi}_{m}$ at a CM point $z$ associated to a quartic CM number field $K$ with totally real subfield $F$ generates the 'CM class field' $H_{\tilde{K}}$ of the reflex field $\tilde{K}$ (see Section 6 for precise definitions). In particular, we show that it generates this CM class field when the CM value $\tilde{\Psi}_{m}(\mathcal{C} \mathcal{M}(K))$, the evaluation of $\tilde{\Psi}_{m}$ at the $\mathrm{CM}$ cycle $\mathcal{C M}(K)$ corresponding to $K$, is not an odd power in $F$ (Corollary 6.3). We also prove the following theorem (see Theorem 6.6 for details).

Theorem 1.2. Let $F=\mathbb{Q}(\sqrt{p})$ be a fixed real quadratic field with $p \equiv 1 \bmod 4$ prime. Then there are constants $c_{1}, c_{2}$ and $d>0$ such that for any $C M$ quartic field $K$ of discriminant $d_{K}=p^{2} q$ with $q \equiv 1 \bmod 4$ prime, and a CM point $z$ in the Hilbert modular surface $X=S L_{2}\left(\mathcal{O}_{F}\right) \backslash \mathbb{H}^{2}$ of $C M$ type $(K, \Phi)$ by $\mathcal{O}_{K}$, one has:

(i) $M\left(\tilde{\Psi}_{1}(z), \tilde{\Psi}_{2}(z)\right)$ is an unramified abelian extension of $M$, where $M$ is the smallest Galois extension of $\mathbb{Q}$ containing $K$.

(ii) Let $H_{\tilde{K}}$ be the 'CM class field' of the reflex field $\tilde{K}$, and let $L_{K}=M H_{\tilde{K}}$. Then $M\left(\tilde{\Psi}_{1}(z), \tilde{\Psi}_{2}(z)\right)$ is a subfield of $L_{K}$ with bounded index $\left[L_{K}: M\left(\tilde{\Psi}_{1}(z), \tilde{\Psi}_{2}(z)\right)\right] \leq d$.

(iii) One has $c_{1} \sqrt{q} \leq\left[M\left(\tilde{\Psi}_{1}(z), \tilde{\Psi}_{2}(z)\right): M\right] \leq c_{2} \sqrt{q}$.

In [BY] the authors derived a formula for the values of (untwisted) Borcherds products (in the sense of $[\mathrm{BB}$ ) at $\mathrm{CM}$ cycles $\mathcal{C} \mathcal{M}(K)$. It would be very interesting to obtain an analogous formula for the CM values of the twisted Borcherds products of Theorem 1.1. In fact one can ask if it is possible to modify the proof of [BY] to give such a result. One key ingredient of the proof - the relation between Borcherds products and automorphic Green functions - is already worked out in the present paper. However, in the second main step of the argument it is not clear at all how the function $\psi$ on the lattice $L^{0}$ defined in (3.5) translates to some natural function on the reflex field $\tilde{K}$.

In Section 7 we study some examples in the special case that $F=\mathbb{Q}(\sqrt{5})$. We write $\tilde{\Psi}_{1}$ and $\tilde{\Psi}_{2}$ in terms of the generators of the ring of symmetric Hilbert modular forms of even weight given by Gundlach $[\mathrm{Gu}$. This can be used to compute the Fourier expansions explicitly, which in turn can be employed to compute some CM values (mainly) numerically. For instance, for the CM point $z_{0}=\left(\zeta_{5}, \zeta_{5}^{2}\right)$ (where $\zeta_{5}=e^{2 \pi i / 5}$ ) corresponding to the cyclic CM extension $K=\mathbb{Q}\left(\zeta_{5}\right)$ of $F=\mathbb{Q}(\sqrt{5})$ we find that

$$
\tilde{\Psi}_{1}\left(z_{0}\right)=\frac{156973921227+70200871784 \sqrt{5}}{156973921227-70200871784 \sqrt{5}}=\frac{\omega^{27} \cdot\left(4+\omega^{\prime}\right)^{5} \cdot\left(5+\omega^{\prime}\right)^{5}}{\omega^{\prime 27} \cdot(4+\omega)^{5} \cdot(5+\omega)^{5}},
$$

where $\omega=\frac{1+\sqrt{5}}{2}$. By means of the results of [BY] we derive a heuristic how the CM values of twisted Borcherds products should look like. In particular, we obtain a conjecture on 
the prime ideals $\mathfrak{l} \subset \mathcal{O}_{F}$ at which the $\mathrm{CM}$ value of a twisted Borcherds product can have non-zero order (see Conjecture [7.1). The same phenomenon as in GZ and [BY] should happen: Such prime ideals should be of small norm.

Finally, in Section 8, we list some open problems for further research. For instance, in all the examples we computed it turned out that $\tilde{\Psi}_{1}\left(\mathcal{C} \mathcal{M}\left(K, \Phi, \mathcal{O}_{F}\right)\right)$ belongs to the field $F$. Moreover, if $K / \mathbb{Q}$ is non-Galois then $\tilde{\Psi}_{1}\left(\mathcal{C M}\left(K, \Phi, \mathcal{O}_{F}\right)\right)$ is square-free. According to Corollary 6.3, this implies that for $z \in \mathcal{C M}\left(K, \Phi, \mathcal{O}_{F}\right)$ the $\mathrm{CM}$ value $\tilde{\Psi}_{1}(z)$ generates the class field $L_{K}$ over $M$. It is an interesting question whether this is a general phenomenon.

We mention that J. Rouse has used Theorem 1.1 to determine the Fourier coefficients of modular functions $f \in \mathbb{Z}[j]$ in terms of traces of singular moduli [Ro].

We thank Heike Hippauf and Sebastian Mayer for their valuable help with the computations in Section 7. Moreover, we thank Lev Borisov and Don Zagier for interesting and useful discussions, and thank Shou-Wu Zhang for bringing to our attention his equidistribution theorem, which is needed in the proof of Theorem 6.6.

\section{Non-holomorphic Poincaré SERIES}

Here we consider non-holomorphic Poincare series of weight 0 . The results of this section are known. We state them for completeness and to fix the notation. For details we refer to [He, $\mathrm{Ni}$, Br2].

Let $I_{\nu}(z)$ and $K_{\nu}(z)$ be the usual modified Bessel functions as in $\mathrm{AbSt} \S 10$. For convenience we put for $s \in \mathbb{C}$ and $y \in \mathbb{R} \backslash\{0\}$ :

$$
\begin{aligned}
\mathcal{I}_{s}(y) & =\sqrt{\frac{\pi|y|}{2}} I_{s-1 / 2}(|y|), \\
\mathcal{K}_{s}(y) & =\sqrt{\frac{2|y|}{\pi}} K_{s-1 / 2}(|y|) .
\end{aligned}
$$

The functions $\mathcal{I}_{s}(y)$ and $\mathcal{K}_{s}(y)$ are holomorphic in $s$. At $s=1$ they have the special values

$$
\begin{aligned}
\mathcal{I}_{1}(y) & =\sinh (|y|), \\
\mathcal{K}_{1}(y) & =e^{-|y|}, \\
2 \mathcal{I}_{1}(y)+\mathcal{K}_{1}(y) & =e^{|y|} .
\end{aligned}
$$

The full elliptic modular group $\Gamma^{\prime}=\mathrm{SL}_{2}(\mathbb{Z})$ acts on the upper complex half plane $\mathbb{H}=$ $\{\tau \in \mathbb{C} ; \Im(\tau)>0\}$ by linear fractional transformations. We write $\Gamma_{\infty}^{\prime}=\left\{\left(\begin{array}{cc}1 & n \\ 0 & 1\end{array}\right) ; n \in \mathbb{Z}\right\}$. As usual we abbreviate $e(x)=e^{2 \pi i x}$.

For a positive integer $m$ we define the Poincaré series of weight 0 and index $m$ by

$$
F_{m}(\tau, s)=\sum_{\gamma \in \Gamma_{\infty}^{\prime} \backslash \Gamma^{\prime}} \mathcal{I}_{s}(2 \pi m \Im(\gamma \tau)) e(-m \Re(\gamma \tau))
$$

where $\tau=x+i y \in \mathbb{H}$ and $s \in \mathbb{C}$ with $\Re(s)>1$. It converges normally for $\Re(s)>1$ and defines a $\Gamma^{\prime}$-invariant function on $\mathbb{H}$. It is an eigenfunction of the hyperbolic Laplacian with eigenvalue $s(s-1)$. 
Theorem 2.1. The Poincaré series $F_{m}(\tau, s)$ has the Fourier expansion

$$
\begin{aligned}
F_{m}(\tau, s)= & \left(2 \mathcal{I}_{s}(2 \pi m y)+\mathcal{K}_{s}(2 \pi m y)\right) e(-m x) \\
& +b_{m}(0, s) y^{1-s}+\sum_{n \in \mathbb{Z} \backslash\{0\}} b_{m}(n, s) \mathcal{K}_{s}(2 \pi n y) e(n x),
\end{aligned}
$$

where

$$
b_{m}(n, s)= \begin{cases}2 \pi\left|\frac{m}{n}\right|^{1 / 2} \sum_{c=1}^{\infty} H_{c}(m, n) I_{2 s-1}\left(\frac{4 \pi}{c} \sqrt{|m n|}\right), & n>0 \\ \frac{4 \pi^{1+s} m^{s}}{(2 s-1) \Gamma(s)} \sum_{c=1}^{\infty} c^{1-2 s} H_{c}(m, 0), & n=0 \\ -\delta_{-m, n}+2 \pi\left|\frac{m}{n}\right|^{1 / 2} \sum_{c=1}^{\infty} H_{c}(m, n) J_{2 s-1}\left(\frac{4 \pi}{c} \sqrt{|m n|}\right), & n<0 .\end{cases}
$$

Here $H_{c}(m, n)$ denotes the Kloosterman sum

$$
H_{c}(m, n)=\frac{1}{c} \sum_{d(c)^{*}} e\left(\frac{n d-m \bar{d}}{c}\right),
$$

where the sum runs through the multiplicative group $(\mathbb{Z} / c \mathbb{Z})^{*}$ and $\bar{d}$ denotes the multiplicative inverse of $d$. Moreover, $J_{\nu}(z)$ and $I_{\nu}(z)$ are the usual Bessel functions as defined in AbSt $\S 9$.

Proof. This is a special case of [Br2] Theorem 1.9. See also [Ni] or [He].

Notice that $H_{c}(m, n)=H_{c}(n, m)$.

Proposition 2.2. The constant term of $F_{m}(\tau, s)$ is equal to

$$
b_{m}(0, s)=\frac{4 \pi}{(2 s-1)} \frac{\sigma_{m}(2 s-1)}{\pi^{-s} \Gamma(s) \zeta(2 s)} .
$$

Here $\zeta(s)$ denotes the Riemann zeta function and $\sigma_{m}(s)$ the divisor sum

$$
\sigma_{m}(s)=m^{(1-s) / 2} \sum_{d \mid m} d^{s} .
$$

Proof. By Theorem 2.1 we have

$$
b_{m}(0, s)=\frac{4 \pi^{1+s} m^{s}}{(2 s-1) \Gamma(s)} \sum_{c=1}^{\infty} c^{-2 s} \sum_{d(c)^{*}} e\left(\frac{m d}{c}\right) .
$$

If we insert the formula for the Ramanujan sum (see Ap Chapter 8.3),

$$
\sum_{d(c)^{*}} e\left(\frac{m d}{c}\right)=\sum_{a \mid(c, m)} \mu(c / a) a
$$


where $\mu$ is the Moebius function, we obtain

$$
\begin{aligned}
b_{m}(0, s) & =\frac{4 \pi^{1+s} m^{s}}{(2 s-1) \Gamma(s)} \sum_{a \mid m} \sum_{\substack{c=1 \\
a \mid c}}^{\infty} c^{-2 s} \mu(c / a) a \\
& =\frac{4 \pi^{1+s}}{(2 s-1) \Gamma(s) \zeta(2 s)} m^{s} \sum_{a \mid m} a^{1-2 s} \\
& =\frac{4 \pi^{1+s} \sigma_{m}(2 s-1)}{(2 s-1) \Gamma(s) \zeta(2 s)} .
\end{aligned}
$$

This proves the Proposition.

Recall that a weakly holomorphic modular form for $\Gamma^{\prime}$ is a meromorphic modular form for $\Gamma^{\prime}$ which is holomorphic outside the cusp $\infty$. In particular, the space of weakly holomorphic modular forms for $\Gamma^{\prime}$ of weight 0 is $\mathbb{C}[j]$, the polynomial ring in the $j$-function.

Theorem 2.3. The special value $F_{m}(\tau, 1)$ has the Fourier expansion

$$
F_{m}(\tau, 1)=q^{-m}+\sum_{n \geq 0} b_{m}(n, 1) q^{n}
$$

where $q=e^{2 \pi i \tau}$ as usual. In particular, $F_{m}(\tau, 1)$ is the unique weakly holomorphic modular form of weight 0 for $\Gamma^{\prime}$ whose Fourier expansion starts $q^{-m}+b_{m}(0,1)+O(q)$.

Proof. If we insert the special values (2.3) and (2.4) in the Fourier expansion given in Theorem 2.3, we find

$$
F_{m}(\tau, 1)=q^{-m}+\sum_{n \geq 0} b_{m}(n, 1) q^{n}+\sum_{n<0} b_{m}(n, 1) e(n \bar{\tau})
$$

This implies that

$$
\overline{\frac{\partial}{\partial \bar{\tau}} F_{m}(\tau, 1)}=-2 \pi i \sum_{n<0} \overline{b_{m}(n, 1)} n e(-n \tau)
$$

is a holomorphic modular form of weight 2 for $\Gamma^{\prime}$ and therefore has to vanish identically. We obtain the assertion.

Remark 2.4. We have $b_{m}(0,1)=24 \sigma_{m}(1)$.

\section{Hilbert modular surfaces and Hirzebruch-Zagier divisors}

Let $p \equiv 1(\bmod 4)$ be a prime and consider the real quadratic field $F=\mathbb{Q}(\sqrt{p})$. We write $\mathcal{O}_{F}$ for the ring of integers in $F$ and $\partial_{F}=(\sqrt{p})$ for the different ideal. Moreover, we write $\epsilon_{p}(x)=\left(\frac{x}{p}\right)$ for the quadratic character of $F$. The conjugation in $F$ is denoted by $x \mapsto x^{\prime}$ and the norm of $x \in F$ by $\mathrm{N}(x)=x x^{\prime}$.

The Hilbert modular group $\Gamma=\mathrm{SL}_{2}\left(\mathcal{O}_{F}\right)$ acts on $\mathbb{H}^{2}$ in the usual way. We denote by $X=\Gamma \backslash \mathbb{H}^{2}$ the Hilbert modular surface associated to $F$. We will use $z=\left(z_{1}, z_{2}\right)$ as a standard variable on $\mathbb{H}^{2}$ and write $z_{1}=x_{1}+i y_{1}, z_{2}=x_{2}+i y_{2}$ for the decomposition in real 
and imaginary parts. Recall that a Hilbert modular form $H\left(z_{1}, z_{2}\right)$ is called symmetric if $H\left(z_{1}, z_{2}\right)=H\left(z_{2}, z_{1}\right)$. It is called skew-symmetric if $H\left(z_{1}, z_{2}\right)=-H\left(z_{2}, z_{1}\right)$.

It is well known that the Hilbert modular group can also be viewed as a discrete subgroup of the orthogonal group of the rational quadratic space

$$
V=\left\{M \in \operatorname{Mat}_{2}(F) ;{ }^{t} M=M^{\prime}\right\}=\left\{\left(\begin{array}{cc}
a & \lambda \\
\lambda^{\prime} & b
\end{array}\right) ; a, b \in \mathbb{Q}, \lambda \in F\right\},
$$

equipped with the quadratic form $M \mapsto \operatorname{det}(M)$. Here ${ }^{t} M$ is the transpose of $M$. The group $\mathrm{SL}_{2}(F)$ acts on $V$ via

$$
\gamma \cdot M=\gamma^{\prime} M^{t} \gamma
$$

leaving the quadratic form invariant. The lattice

$$
L=\left\{\left(\begin{array}{cc}
a & \lambda \\
\lambda^{\prime} & b
\end{array}\right) \in V ; a, b \in \mathbb{Z}, \lambda \in \partial_{F}^{-1}\right\}
$$

is stable under the action of $\Gamma$. Recall that $M \in L$ is called primitive, if $\frac{1}{d} M \notin L$ for every integer $d>1$.

If $M=\left(\begin{array}{cc}a & \lambda \\ \lambda^{\prime} & b\end{array}\right)$ is a vector in $L$, we let

$$
M^{\perp}=\left\{\left(z_{1}, z_{2}\right) \in \mathbb{H}^{2} ; \quad a z_{1} z_{2}+\lambda z_{1}+\lambda^{\prime} z_{2}+b=0\right\}
$$

be the corresponding analytic divisor on $\mathbb{H}^{2}$. If $m$ is a positive integer, then

$$
F_{m}=\sum_{\substack{M \in L /\{ \pm 1\} \text { primitive } \\ \operatorname{det}(M)=m / p}} M^{\perp}
$$

is a $\Gamma$-invariant divisor on $\mathbb{H}^{2}$, which descends to an algebraic divisor on $X$, also denoted by $F_{m}$. It is well known that the Hirzebruch-Zagier divisors $T_{m}$ on $X$ can be written as $T_{m}=\sum_{d^{2} \mid m} F_{m / d^{2}}$ (see $\mathrm{Ge},[\mathrm{HZ}]$ ).

The divisor $F_{m}$ on $X$ is irreducible if and only if $p^{2} \nmid m$. If $p^{2} \mid m$ then it decomposes into two irreducible components $F_{m}=F_{m}^{+}+F_{m}^{-}$. To distinguish these components we define a function on lattice vectors $M=\left(\begin{array}{cc}a & \lambda \\ \lambda^{\prime} & b\end{array}\right) \in L$ of norm $a b-\lambda \lambda^{\prime} \in p \mathbb{Z}$ divisible by $p$ by

$$
\psi(M)= \begin{cases}\left(\frac{a}{p}\right), & \text { if } p \nmid a, \\ \left(\frac{b}{p}\right), & \text { if } p \nmid b, \\ 0, & \text { if } p \mid(a, b) .\end{cases}
$$

The following lemma shows that $\psi$ is well defined.

Lemma 3.1. Let $M=\left(\begin{array}{cc}a & \lambda \\ \lambda^{\prime} & b\end{array}\right) \in L$ be a p-primitive vector (i.e. $\left.\frac{1}{p} M \notin L\right)$ and assume that $a b-\lambda \lambda^{\prime}=n p$ for some $n \in \mathbb{Z}$. Then $\left(\frac{a}{p}\right)+\left(\frac{b}{p}\right) \neq 0$.

Proof. First we notice that the hypothesis implies $p \nmid(a, b)$. This immediately yields the assertion if $p \mid a$, or $p \mid b$, respectively. So we may assume that $p \nmid a$ and $p \nmid b$. We have to show that $\left(\frac{a}{p}\right)=\left(\frac{b}{p}\right)$. If we write $\lambda=\frac{1}{2}(c+d \sqrt{p})$ with $c, d \in \mathbb{Z}$ we find that

$$
4 a b=c^{2}-p d^{2}+4 n p \equiv \alpha^{2} \quad(\bmod p) .
$$

This implies the assertion. 
The function $\psi$ has the following genus character interpretation. Assume that $M=$ $\left(\begin{array}{ll}a & \lambda \\ \lambda^{\prime} & b\end{array}\right) \in L$ and $a b-\lambda \lambda^{\prime} \in p \mathbb{Z}$. Then $\lambda$ actually belongs to $\mathcal{O}_{F}$ and we may write $\lambda=$ $\frac{1}{2}(c+d \sqrt{p})$ with $c, d \in \mathbb{Z}$. We have

$$
\left(\begin{array}{cc}
a & \lambda \\
\lambda^{\prime} & b
\end{array}\right) \equiv\left(\begin{array}{cc}
a & c / 2 \\
c / 2 & b
\end{array}\right) \quad\left(\bmod \partial_{F}\right)
$$

and the latter matrix defines a binary integral quadratic form $Q=[a, c, b]$ of discriminant $c^{2}-4 a b$ divisible by $p$. Recall that on binary integral quadratic forms of discriminant divisible by $p$ we have a genus character $\chi_{p}$ which is defined as follows:

$$
\chi_{p}(Q)= \begin{cases}\left(\frac{n}{p}\right), & \text { if } p \nmid Q \text { and }(n, p)=1 \text { and } Q \text { represents } n, \\ 0, & \text { if } p \mid Q .\end{cases}
$$

This definition does not depend on the choice of $n$. If the quadratic form $Q$ corresponds to $M$ as in (3.6), then $\psi(M)=\chi_{p}(Q)$.

One easily verifies that the value of $\psi(M)$ only depends on the $\Gamma$-orbit of $M$. In fact, it is well known that the components of $F_{m p^{2}}$ are distinguished by the values of the function $\psi$ (see [Za2, Ge Chapter V.3).

Definition 3.2. For a positive integer $m$ we define the twisted Hirzebruch-Zagier divisor of index $m$ by

$$
\tilde{T}_{m}=\sum_{\substack{M \in L /\{ \pm 1\} \\ \operatorname{det}(M)=m p}} \psi(M) \cdot M^{\perp}
$$

For instance, if $m$ is square-free then $\tilde{T}_{m}=F_{m p^{2}}^{+}-F_{m p^{2}}^{-}$.

\section{Automorphic Green functions}

Let $m$ be a positive integer. Following [Br1] we define the automorphic Green function corresponding to $\tilde{T}_{m}$ by

$$
\tilde{\Phi}_{m p^{2}}\left(z_{1}, z_{2}, s\right)=\sum_{\substack{a \\
\left(\begin{array}{c}
a \\
\lambda^{\prime} \\
b
\end{array}\right) \in L \\
a b-\mathrm{N}(\lambda)=m p}} \psi\left(\begin{array}{cc}
a & \lambda \\
\lambda^{\prime} & b
\end{array}\right) Q_{s-1}\left(1+\frac{\left|a z_{1} z_{2}+\lambda z_{1}+\lambda^{\prime} z_{2}+b\right|^{2}}{2 y_{1} y_{2} m p}\right)
$$

where $Q_{s-1}(t)$ is the Legendre function of the second kind defined by (cf. [AbSt] 88 )

$$
Q_{s-1}(t)=\int_{0}^{\infty}\left(t+\sqrt{t^{2}-1} \cosh v\right)^{-s} d v \quad(t>1, \Re(s)>0) .
$$

The sum converges normally for $\left(z_{1}, z_{2}\right) \in \mathbb{H}^{2} \backslash \tilde{T}_{m}$ and $s \in \mathbb{C}$ with $\Re(s)>1$. We will continue it to a neighborhood of $s=1$ by computing the Fourier expansion. To this end we write

$$
\tilde{\Phi}_{m p^{2}}\left(z_{1}, z_{2}, s\right)=\tilde{\Phi}_{m p^{2}}^{0}\left(z_{1}, z_{2}, s\right)+2 \sum_{a=1}^{\infty} \tilde{\Phi}_{m p^{2}}^{a}\left(z_{1}, z_{2}, s\right)
$$


with

$$
\tilde{\Phi}_{m p^{2}}^{a}\left(z_{1}, z_{2}, s\right)=\sum_{\substack{b \in \mathbb{Z} \\
\lambda \in \partial_{F}^{-1} \\
a b-\mathrm{N}(\lambda)=m p}} \psi\left(\begin{array}{cc}
a & \lambda \\
\lambda^{\prime} & b
\end{array}\right) Q_{s-1}\left(1+\frac{\left|a z_{1} z_{2}+\lambda z_{1}+\lambda^{\prime} z_{2}+b\right|^{2}}{2 y_{1} y_{2} m p}\right) .
$$

Note that the partial sums $\tilde{\Phi}_{m p^{2}}^{a}\left(z_{1}, z_{2}, s\right)$ converge normally for $\Re(s)>1 / 2$.

For $a=0$ we have

$$
\tilde{\Phi}_{m p^{2}}^{0}\left(z_{1}, z_{2}, s\right)=\sum_{\substack{b \in \mathbb{Z} \\ \lambda \in \partial_{F}^{-1} \\ \mathrm{~N}(\lambda)=-m p}} \epsilon_{p}(b) Q_{s-1}\left(1+\frac{\left|\lambda z_{1}+\lambda^{\prime} z_{2}+b\right|^{2}}{2 y_{1} y_{2} m p}\right) .
$$

If $a$ is a positive integer coprime to $p$, then

$$
\begin{aligned}
\tilde{\Phi}_{m p^{2}}^{a}\left(z_{1}, z_{2}, s\right) & =\epsilon_{p}(a) \sum_{\substack{b \in \mathbb{Z} \\
\lambda \in \partial_{F}^{-1} \\
a b-\mathrm{N}(\lambda)=m p}} Q_{s-1}\left(1+\frac{\left|a z_{1} z_{2}+\lambda z_{1}+\lambda^{\prime} z_{2}+b\right|^{2}}{2 y_{1} y_{2} m p}\right) \\
& =\epsilon_{p}(a) \Phi_{m p^{2}}^{a}\left(z_{1}, z_{2}, s\right),
\end{aligned}
$$

where $\Phi_{m p^{2}}^{a}\left(z_{1}, z_{2}, s\right)$ is the function defined in Br1] (see $\S 3$ equation (16)). If $a$ is divisible by $p$, then

$$
\tilde{\Phi}_{m p^{2}}^{a}\left(z_{1}, z_{2}, s\right)=\sum_{\substack{b \in \mathbb{Z} \\ \lambda \in \partial_{F}^{-1} \\ a b-\mathrm{N}(\lambda)=m p}} \epsilon_{p}(b) Q_{s-1}\left(1+\frac{\left|a z_{1} z_{2}+\lambda z_{1}+\lambda^{\prime} z_{2}+b\right|^{2}}{2 y_{1} y_{2} m p}\right) .
$$

We compute the Fourier expansion of $\tilde{\Phi}_{m p^{2}}^{a}\left(z_{1}, z_{2}, s\right)$ in these three cases. We put for a positive real number $A$,

$$
H_{s}^{A}\left(z_{1}, z_{2}\right)=\sum_{\theta \in \mathcal{O}} Q_{s-1}\left(1+\frac{\left|\left(z_{1}+\theta\right)\left(z_{2}+\theta^{\prime}\right)+A\right|^{2}}{2 y_{1} y_{2} A}\right),
$$

and denote (for $y_{1} y_{2}>A$ ) the Fourier expansion by

$$
H_{s}^{A}\left(z_{1}, z_{2}\right)=\sum_{\nu \in \mathfrak{D}^{-1}} b_{s}^{A}\left(\nu, y_{1}, y_{2}\right) e\left(\nu x_{1}+\nu^{\prime} x_{2}\right)
$$

Moreover, we let $R\left(m p^{2}\right)$ be a set of representatives for

$$
\begin{aligned}
& \left\{\lambda \in \partial_{F}^{-1} / a \mathcal{O}_{F} ; \quad \mathrm{N}(\lambda \sqrt{p}) \equiv m p^{2} \quad(\bmod a p)\right\} \\
& =\left\{\lambda \in \mathcal{O}_{F} / a \mathcal{O}_{F} ; \quad \mathrm{N}(\lambda) \equiv-m p \quad(\bmod a)\right\} .
\end{aligned}
$$


We start with the case that $a$ is positive and coprime to $p$. Here we can argue as in Br1]. We may write

$$
\begin{aligned}
\tilde{\Phi}_{m p^{2}}^{a}\left(z_{1}, z_{2}, s\right) & =\epsilon_{p}(a) \sum_{\lambda \in R\left(m p^{2}\right)} \sum_{\theta \in \mathcal{O}} Q_{s-1}\left(1+\frac{\left|\left(z_{1}+\theta+\frac{\lambda^{\prime}}{a}\right)\left(z_{2}+\theta^{\prime}+\frac{\lambda}{a}\right)+\frac{m p}{a^{2}}\right|^{2}}{2 y_{1} y_{2} m p / a^{2}}\right) \\
& =\sum_{\nu \in \partial_{F}^{-1}} \epsilon_{p}(a) \sum_{\lambda \in R\left(m p^{2}\right)} e\left(\frac{\operatorname{tr}(\nu \lambda)}{a}\right) b_{s}^{m p / a^{2}}\left(\nu, y_{1}, y_{2}\right) e\left(\nu z_{1}+\nu^{\prime} z_{2}\right) .
\end{aligned}
$$

When $a$ is positive and divisible by $p$, one finds in a similar way that

$$
\begin{aligned}
\tilde{\Phi}_{m p^{2}}^{a}\left(z_{1}, z_{2}, s\right) & =\sum_{\lambda \in R\left(m p^{2}\right)} \sum_{\theta \in \mathcal{O}} \epsilon_{p}\left(\frac{\lambda \lambda^{\prime}+m p}{a}\right) Q_{s-1}\left(1+\frac{\left|\left(z_{1}+\theta+\frac{\lambda^{\prime}}{a}\right)\left(z_{2}+\theta^{\prime}+\frac{\lambda}{a}\right)+\frac{m p}{a^{2}}\right|^{2}}{2 y_{1} y_{2} m p / a^{2}}\right) \\
& =\sum_{\nu \in \partial_{F}^{-1}} \sum_{\lambda \in R\left(m p^{2}\right)} \epsilon_{p}\left(\frac{\lambda \lambda^{\prime}+m p}{a}\right) e\left(\frac{\operatorname{tr}(\nu \lambda)}{a}\right) b_{s}^{m p / a^{2}}\left(\nu, y_{1}, y_{2}\right) e\left(\nu z_{1}+\nu^{\prime} z_{2}\right) .
\end{aligned}
$$

If we define

$$
\tilde{G}_{a}\left(m p^{2}, \nu\right)= \begin{cases}\epsilon_{p}(a) \sum_{\lambda \in R\left(m p^{2}\right)} e\left(\frac{\operatorname{tr}(\nu \lambda)}{a}\right), & \text { if } p \nmid a, \\ \sum_{\lambda \in R\left(m p^{2}\right)} \epsilon_{p}\left(\frac{\lambda \lambda^{\prime}+m p}{a}\right) e\left(\frac{\operatorname{tr}(\nu \lambda)}{a}\right), & \text { if } p \mid a,\end{cases}
$$

we may finally write

$$
\tilde{\Phi}_{m p^{2}}\left(z_{1}, z_{2}, s\right)=\tilde{\Phi}_{m p^{2}}^{0}\left(z_{1}, z_{2}, s\right)+2 \sum_{\nu \in \partial_{F}^{-1}}\left[\sum_{a=1}^{\infty} \tilde{G}_{a}\left(m p^{2}, \nu\right) b_{s}^{m p / a^{2}}\left(\nu, y_{1}, y_{2}\right)\right] e\left(\nu x_{1}+\nu^{\prime} x_{2}\right) \text {. }
$$

For $r_{1}, r_{2} \in \mathbb{R}$ we briefly write

$$
\begin{gathered}
\alpha\left(r_{1}, r_{2}\right):=\max \left(\left|r_{1}\right|,\left|r_{2}\right|\right), \\
\beta\left(r_{1}, r_{2}\right):=\min \left(\left|r_{1}\right|,\left|r_{2}\right|\right) .
\end{gathered}
$$

Lemma 4.1. The function $\tilde{\Phi}_{m p^{2}}^{0}\left(z_{1}, z_{2}, s\right)$ has the Fourier expansion

$$
\begin{aligned}
\tilde{\Phi}_{m p^{2}}^{0}\left(z_{1}, z_{2}, s\right)=2 \sqrt{p} \sum_{\substack{\lambda \in \partial_{F}^{-1} \\
\mathrm{~N}(\lambda)=-m / p}} \sum_{n \geq 1} \frac{\epsilon_{p}(n)}{n} \mathcal{I}_{s}\left(2 \pi n \beta\left(\lambda y_{1}, \lambda^{\prime} y_{2}\right)\right) \\
\quad \times \mathcal{K}_{s}\left(2 \pi n \alpha\left(\lambda y_{1}, \lambda^{\prime} y_{2}\right)\right) e\left(n \lambda x_{1}+n \lambda^{\prime} x_{2}\right)
\end{aligned}
$$


Proof. By definition we have

$$
\begin{aligned}
\tilde{\Phi}_{m p^{2}}^{0}\left(z_{1}, z_{2}, s\right)= & \sum_{\substack{b \in \mathbb{Z}, \lambda \in \partial_{F} \\
\lambda \lambda^{\prime}=-m p}} \epsilon_{p}(b) Q_{s-1}\left(1+\frac{\left|\lambda z_{1}+\lambda^{\prime} z_{2}+b\right|^{2}}{2 y_{1} y_{2} m p}\right) \\
= & \sum_{\substack{b(p), \lambda \in \partial_{F}^{-1} \\
\lambda \lambda^{\prime}=-m / p}} \epsilon_{p}(b) \sum_{b^{\prime} \in \mathbb{Z}} Q_{s-1}\left(1+\frac{\left|\lambda z_{1}+\lambda^{\prime} z_{2}+b^{\prime}+b / p\right|^{2}}{2 y_{1} y_{2} m / p}\right) .
\end{aligned}
$$

We find that

$$
\tilde{\Phi}_{m p^{2}}^{0}\left(z_{1}, z_{2}, s\right)=\sum_{b(p)} \sum_{\substack{\lambda \in \partial_{F}^{-1} \\ \lambda \lambda^{\prime}=-m / p}} \epsilon_{p}(b) h_{\alpha\left(\lambda y_{1}, \lambda^{\prime} y_{2}\right), \beta\left(\lambda y_{1}, \lambda^{\prime} y_{2}\right)}\left(\lambda x_{1}+\lambda^{\prime} x_{2}+b / p\right),
$$

where

$$
h_{\alpha, \beta}(x)=\sum_{b \in \mathbb{Z}} Q_{s-1}\left(\frac{(x+b)^{2}+\alpha^{2}+\beta^{2}}{2 \alpha \beta}\right) .
$$

By Br1] Lemma 1, for $\alpha>\beta>0$, the function $h_{\alpha, \beta}(x)$ has the Fourier expansion

$$
h_{\alpha, \beta}(x)=\frac{2 \pi}{2 s-1} \alpha^{1-s} \beta^{s}+\sum_{n \in \mathbb{Z}-\{0\}} \frac{1}{|n|} \mathcal{I}_{s}(2 \pi n \beta) \mathcal{K}_{s}(2 \pi n \alpha) e(n x) .
$$

Inserting this into (4.8), we obtain the assertion.

We recall the following lemma from $[\mathrm{Br} 1$ :

Lemma 4.2. Let $y_{1} y_{2}>A>0$. The function $H_{s}^{A}\left(z_{1}, z_{2}\right)$ defined by 4.4 has the Fourier expansion

$$
H_{s}^{A}\left(z_{1}, z_{2}\right)=\sum_{\nu \in \partial_{F}^{-1}} b_{s}^{A}\left(\nu, y_{1}, y_{2}\right) e\left(\nu x_{1}+\nu^{\prime} x_{2}\right)
$$

with

$$
\begin{array}{ll}
b_{s}^{A}\left(0, y_{1}, y_{2}\right) & =\frac{\pi \Gamma(s-1 / 2)^{2}}{2 \sqrt{p} \Gamma(2 s)}(4 A)^{s}\left(y_{1} y_{2}\right)^{1-s}, \\
b_{s}^{A}\left(\nu, y_{1}, y_{2}\right) & =\pi \sqrt{\frac{A}{p\left|\nu \nu^{\prime}\right|}} I_{2 s-1}\left(4 \pi \sqrt{A\left|\nu \nu^{\prime}\right|}\right) \mathcal{K}_{s}\left(2 \pi|\nu| y_{1}\right) \mathcal{K}_{s}\left(2 \pi\left|\nu^{\prime}\right| y_{2}\right), \quad \nu \nu^{\prime}>0, \\
b_{s}^{A}\left(\nu, y_{1}, y_{2}\right) & =\pi \sqrt{\frac{A}{p\left|\nu \nu^{\prime}\right|}} J_{2 s-1}\left(4 \pi \sqrt{A\left|\nu \nu^{\prime}\right|}\right) \mathcal{K}_{s}\left(2 \pi|\nu| y_{1}\right) \mathcal{K}_{s}\left(2 \pi\left|\nu^{\prime}\right| y_{2}\right), \quad \nu \nu^{\prime}<0 .
\end{array}
$$

The following identity of finite exponential sums is crucial for the main result of the present paper. 
Lemma 4.3. Let $a \in \mathbb{N}, m \in \mathbb{Z}$, and $\nu \in \partial_{F}^{-1}$. Then

$$
\frac{1}{a} \tilde{G}_{a}\left(m p^{2}, \nu\right)=\sum_{\substack{r|\nu \\ r| a}} \epsilon_{p}(r) H_{a / r}\left(\frac{p \nu \nu^{\prime}}{r^{2}}, m\right)
$$

where the finite exponential sums $\tilde{G}_{a}(m, \nu)$ resp. $H_{b}(m, n)$ are defined by (4.5) resp. 2.7).

Proof. We follow the proof of the Proposition in Za1] §4. Both sides in (4.9) are clearly periodic in $m$ with period $a$. Therefore it suffices to show that the finite Fourier transforms are equal, i.e., that for every $h(\bmod a)$ we have

$$
\frac{1}{a} \sum_{m(a)} e\left(-\frac{h m}{a}\right) \tilde{G}_{a}\left(m p^{2}, \nu\right)=\sum_{m(a)} e\left(-\frac{h m}{a}\right) \sum_{\substack{r|\nu \\ r| a}} \epsilon_{p}(r) H_{a / r}\left(\frac{p \nu \nu^{\prime}}{r^{2}}, m\right) .
$$

Inserting the definition of $H_{c}(n, m)$ we find that the right hand side equals

$$
\sum_{\substack{r|\nu \\ r| a}} \epsilon_{p}(r) \frac{r}{a} \sum_{d(a / r)^{*}} e\left(\frac{d p \nu \nu^{\prime} / r^{2}}{a / r}\right) \sum_{m(a)} e\left(-m \frac{\bar{d} r+h}{a}\right) .
$$

The sum over $m(a)$ vanishes unless $\bar{d} r+h \equiv 0(\bmod a)$ in which case it equals $a$. But $\bar{d} r+h \equiv 0(\bmod a)$ implies that $h \equiv 0(\bmod r)$ and $\bar{d}+h / r \equiv 0(\bmod a / r)$. Since $\bar{d}$ is coprime to $a / r$ we find that $h / r$ must also be coprime to $a / r$ and consequently $r=(h, a)$.

Thus, denoting $r=(h, a)$ and by $\bar{h}_{1}$ a multiplicative inverse of $h / r$ modulo $a / r$ we obtain that the right hand side of (4.10) equals

$$
\epsilon_{p}(r) r \cdot e\left(-\frac{\bar{h}_{1} p \nu \nu^{\prime} / r^{2}}{a / r}\right)
$$

We now consider the left hand side of (4.10). We first assume that $(p, a)=1$. In this case it is equal to

$$
\begin{aligned}
& \frac{\epsilon_{p}(a)}{a} \sum_{m(a)} \sum_{\substack{\lambda \in \mathcal{O}_{F} / a \mathcal{O}_{F} \\
\lambda \lambda^{\prime}=-m p(a)}} e\left(\frac{\operatorname{tr}(\lambda \nu)-h m}{a}\right) \\
& =\frac{\epsilon_{p}(a)}{a} \sum_{\substack{\lambda \in \mathcal{O}_{F} / a \mathcal{O}_{F}\\
}} e\left(\frac{\operatorname{tr}(\lambda \nu)+h \bar{p} \lambda \lambda^{\prime}}{a}\right) .
\end{aligned}
$$

Here $\bar{p}$ denotes a multiplicative inverse of $p$ modulo $a$. We first observe that the sum vanishes unless $r=(h, a)$ divides $\nu$. This is easily seen by replacing $\lambda \mapsto \lambda+\frac{a}{r} \tau$ for $\tau \in \mathcal{O}_{F}$ in the sum. Therefore, put $r=(h, a)$ and write $h=h_{1} r, a=a_{1} r$, and $\nu=\nu_{1} r$. Then the 
sum becomes

$$
\begin{aligned}
& \frac{\epsilon_{p}(a)}{a} \sum_{\lambda \in \mathcal{O}_{F} / a \mathcal{O}_{F}} e\left(\frac{\operatorname{tr}\left(\lambda \nu_{1}\right)+h_{1} \bar{p} \lambda \lambda^{\prime}}{a_{1}}\right) \\
& =\frac{\epsilon_{p}(a)}{a} r^{2} e\left(-\frac{p \bar{h}_{1} \nu_{1} \nu_{1}^{\prime}}{a_{1}}\right) \sum_{\lambda \in \mathcal{O}_{F} / a_{1} \mathcal{O}_{F}} e\left(\frac{h_{1} \bar{p}\left(\lambda+p \bar{h}_{1} \nu_{1}^{\prime}\right)\left(\lambda^{\prime}+p \bar{h}_{1} \nu_{1}\right)}{a_{1}}\right) \\
& =\frac{\epsilon_{p}(a)}{a_{1}} r e\left(-\frac{p \bar{h}_{1} \nu_{1} \nu_{1}^{\prime}}{a_{1}}\right) \sum_{\lambda \in \mathcal{O}_{F} / a_{1} \mathcal{O}_{F}} e\left(\frac{h_{1} \bar{p} \lambda \lambda^{\prime}}{a_{1}}\right) .
\end{aligned}
$$

The latter sum is computed in Za1] $§ 4$ Lemma 2. Inserting its value

$$
\frac{1}{a_{1}} \sum_{\lambda \in \mathcal{O}_{F} / a_{1} \mathcal{O}_{F}} e\left(\frac{h_{1} \bar{p} \lambda \lambda^{\prime}}{a_{1}}\right)=\epsilon_{p}\left(a_{1}\right)
$$

we finally find that the left hand side of (4.10) is equal to

$$
\epsilon_{p}(r) r \cdot e\left(-\frac{p \bar{h}_{1} \nu \nu^{\prime} / r^{2}}{a / r}\right)
$$

Let us now consider the left hand side of (4.10) in the case that $p \mid a$. Then it is given by

$$
\frac{1}{a} \sum_{m(a)} \sum_{\substack{\lambda \in \mathcal{O}_{F} / a \mathcal{O}_{F} \\ \lambda \lambda^{\prime} \equiv-m p(a)}} \epsilon_{p}\left(\frac{\lambda \lambda^{\prime}+m p}{a}\right) e\left(\frac{\operatorname{tr}(\nu \lambda)-m h}{a}\right) .
$$

We substitute $m=m_{1}+m_{2} a / p$, where $m_{1}$ runs modulo $a / p$ and $m_{2}$ modulo $p$. Moreover, we notice that in the sum over $\lambda$, we actually only sum over $\lambda \in \partial_{F} / a \mathcal{O}_{F}$, since $p \mid a$. Substituting $\lambda \mapsto \sqrt{p} \lambda$, we obtain

$$
\begin{aligned}
& \frac{1}{a} \sum_{\substack{m_{1}(a / p) \\
m_{2}(p)}} \sum_{\substack{\lambda \in \mathcal{O}_{F} / a \partial_{F}^{-1} \\
\lambda \lambda^{\prime} \equiv m_{1}(a / p)}} \epsilon_{p}\left(\frac{-\lambda \lambda^{\prime}+m_{1}}{a / p}+m_{2}\right) e\left(\frac{\operatorname{tr}(\sqrt{p} \lambda \nu)-m_{1} h}{a}\right) e\left(-\frac{h m_{2}}{p}\right) \\
& =\frac{1}{a p} \sum_{m_{2}(p)} \sum_{\lambda \in \mathcal{O}_{F} / a \mathcal{O}_{F}} e\left(\frac{\operatorname{tr}(\sqrt{p} \lambda \nu)-h \lambda \lambda^{\prime}}{a}\right) \epsilon_{p}\left(m_{2}\right) e\left(-\frac{h m_{2}}{p}\right) \\
& =\frac{1}{a \sqrt{p}} \epsilon_{p}(h) \sum_{\lambda \in \mathcal{O}_{F} / a \mathcal{O}_{F}} e\left(\frac{\operatorname{tr}(\sqrt{p} \lambda \nu)-h \lambda \lambda^{\prime}}{a}\right) .
\end{aligned}
$$

Here, in the last line we have inserted the value of the Gauss sum. In particular we see that the latter quantity vanishes if $p \mid h$. If $p \nmid(h, a)$ we see by replacing $\lambda \mapsto \lambda+\frac{a}{r} \tau$ for $\tau \in \mathcal{O}_{F}$ that the latter quantity actually vanishes unless $(h, a)$ divides $\nu$. Therefore, as 
above, we put $r=(h, a)$ and write $h=h_{1} r, a=a_{1} r$, and $\nu=\nu_{1} r$. Then the sum becomes

$$
\begin{aligned}
& \frac{r^{2}}{a \sqrt{p}} \epsilon_{p}(h) \sum_{\lambda \in \mathcal{O}_{F} / a_{1} \mathcal{O}_{F}} e\left(\frac{\operatorname{tr}\left(\sqrt{p} \lambda \nu_{1}\right)-h_{1} \lambda \lambda^{\prime}}{a_{1}}\right) \\
& =\frac{r}{a_{1 \sqrt{p}}} \epsilon_{p}(h) e\left(-\frac{\bar{h}_{1} p \nu_{1} \nu_{1}^{\prime}}{a_{1}}\right) \sum_{\lambda \in \mathcal{O}_{F} / a_{1} \mathcal{O}_{F}} e\left(-\frac{h_{1}\left(\lambda-\left(\bar{h}_{1} \sqrt{p} \nu_{1}\right)^{\prime}\right)\left(\lambda^{\prime}-\left(\bar{h}_{1} \sqrt{p} \nu_{1}\right)^{\prime}\right)^{\prime}}{a_{1}}\right) \\
& =\frac{r}{a_{1 \sqrt{p}}} \epsilon_{p}(h) e\left(-\frac{\bar{h}_{1} p \nu \nu^{\prime} / r^{2}}{a / r}\right) \sum_{\lambda \in \mathcal{O}_{F} / a_{1} \mathcal{O}_{F}} e\left(-\frac{h_{1} \lambda \lambda^{\prime}}{a_{1}}\right) .
\end{aligned}
$$

By Za1] $§ 4$ Lemma 2 (noting that $p \nmid r$ implies $p \mid a_{1}$ ) we have

$$
\frac{1}{a_{1}} \sum_{\lambda \in \mathcal{O}_{F} / a_{1} \mathcal{O}_{F}} e\left(-\frac{h_{1} \lambda \lambda^{\prime}}{a_{1}}\right)=\epsilon_{p}\left(h_{1}\right) \sqrt{p} .
$$

We finally find that left hand side of (4.10) is equal to

$$
\epsilon_{p}(r) r \cdot e\left(-\frac{p \bar{h}_{1} \nu \nu^{\prime} / r^{2}}{a / r}\right) .
$$

This concludes the proof of the Lemma.

We are now ready to compute the Fourier expansion of $\tilde{\Phi}_{m p^{2}}\left(z_{1}, z_{2}, s\right)$ in terms of the coefficients of the Poincaré series $F_{m}(\tau, s)$.

Theorem 4.4. The automorphic Green function $\tilde{\Phi}_{m p^{2}}\left(z_{1}, z_{2}, s\right)$ associated to $\tilde{T}_{m}$ has the Fourier expansion

$$
\begin{aligned}
& \tilde{\Phi}_{m p^{2}}\left(z_{1}, z_{2}, s\right) \\
& =\left(\frac{p}{\pi}\right)^{s-1 / 2} \Gamma(s-1 / 2) L\left(2 s-1, \epsilon_{p}\right) b_{m}(0, s)\left(y_{1} y_{2}\right)^{1-s} \\
& +\sqrt{p} \sum_{\substack{\nu \in \partial_{F}^{-1} \\
\nu \nu^{\prime} \neq 0}} \sum_{n \geq 1} \frac{\epsilon_{p}(n)}{n} b_{m}\left(p \nu \nu^{\prime}, s\right) \mathcal{K}_{s}\left(2 \pi n \nu y_{1}\right) \mathcal{K}_{s}\left(2 \pi n \nu^{\prime} y_{2}\right) e\left(\nu n x_{1}+\nu^{\prime} n x_{2}\right) \\
& +\sqrt{p} \sum_{\substack{\lambda \in \partial_{F}^{-1} \\
\mathrm{~N}(\lambda)=-m / p}} \sum_{n \geq 1} \frac{\epsilon_{p}(n)}{n}\left(2 \mathcal{I}_{s}\left(2 \pi n \beta\left(\lambda y_{1}, \lambda^{\prime} y_{2}\right)\right)+\mathcal{K}_{s}\left(2 \pi n \beta\left(\lambda y_{1}, \lambda^{\prime} y_{2}\right)\right)\right) \\
& \times \mathcal{K}_{s}\left(2 \pi n \alpha\left(\lambda y_{1}, \lambda^{\prime} y_{2}\right)\right) e\left(n \lambda x_{1}+n \lambda^{\prime} x_{2}\right) .
\end{aligned}
$$

If converges normally for $y_{1} y_{2}>m p$. Here $b_{m}(n, s)$ denote the Fourier coefficients of the Poincaré series $F_{m}(\tau, s)$, and $L\left(s, \epsilon_{p}\right)$ the Dirichlet L-function corresponding to $\epsilon_{p}$. 
Proof. We use (4.6), Lemma 4.1, and Lemma 4.2 to compute the Fourier expansion. The constant term is given by

$$
\begin{aligned}
& 2 \sum_{a=1}^{\infty} \tilde{G}_{a}\left(m p^{2}, 0\right) b_{s}^{m p / a^{2}}\left(0, y_{1}, y_{2}\right) \\
& =\frac{\pi \Gamma(s-1 / 2)^{2}}{\sqrt{p} \Gamma(2 s)}(4 m p)^{s}\left(y_{1} y_{2}\right)^{1-s} \sum_{a=1}^{\infty} \tilde{G}_{a}\left(m p^{2}, 0\right) a^{-2 s} .
\end{aligned}
$$

In view of Lemma 4.3 this is equal to

$$
\begin{aligned}
& \frac{\pi \Gamma(s-1 / 2)^{2}}{\sqrt{p} \Gamma(2 s)}(4 m p)^{s}\left(y_{1} y_{2}\right)^{1-s} \sum_{a=1}^{\infty} \sum_{r \mid a} \epsilon_{p}(r) H_{a / r}(m, 0) a^{1-2 s} \\
& =\frac{\pi \Gamma(s-1 / 2)^{2}}{\sqrt{p} \Gamma(2 s)}(4 m p)^{s}\left(y_{1} y_{2}\right)^{1-s} \sum_{c=1}^{\infty} \sum_{r=1}^{\infty} \epsilon_{p}(r) H_{c}(m, 0)(c r)^{1-2 s} \\
& =\frac{\pi \Gamma(s-1 / 2)^{2}}{\sqrt{p} \Gamma(2 s)}(4 m p)^{s}\left(y_{1} y_{2}\right)^{1-s} L\left(2 s-1, \epsilon_{p}\right) \sum_{c=1}^{\infty} H_{c}(m, 0) c^{1-2 s} .
\end{aligned}
$$

By means of the formula for the constant coefficient $b_{m}(0, s)$ of $F_{m}(\tau, s)$ and the duplication formula $\Gamma(s) \Gamma\left(s+\frac{1}{2}\right)=2^{1-2 s} \sqrt{\pi} \Gamma(2 s)$ we see that the constant term equals:

$$
\left(\frac{p}{\pi}\right)^{s-1 / 2} \Gamma(s-1 / 2) L\left(2 s-1, \epsilon_{p}\right) b_{m}(0, s)\left(y_{1} y_{2}\right)^{1-s} .
$$

We now consider the $\nu$-th Fourier coefficient of $\tilde{\Phi}_{m p^{2}}\left(z_{1}, z_{2}, s\right)$ for $\nu \in \partial_{F}$ with $\nu \nu^{\prime}>0$. It is given by

$$
\begin{aligned}
& 2 \sum_{a=1}^{\infty} \tilde{G}_{a}\left(m p^{2}, \nu\right) b_{s}^{m p / a^{2}}\left(\nu, y_{1}, y_{2}\right) \\
& =2 \pi \sum_{a=1}^{\infty} \frac{1}{a} \tilde{G}_{a}\left(m p^{2}, \nu\right) \sqrt{\frac{m}{\nu \nu^{\prime}}} I_{2 s-1}\left(\frac{4 \pi}{a} \sqrt{m p\left|\nu \nu^{\prime}\right|}\right) \mathcal{K}_{s}\left(2 \pi \nu y_{1}\right) \mathcal{K}_{s}\left(2 \pi \nu^{\prime} y_{2}\right) .
\end{aligned}
$$

In view of Lemma 4.3 this is equal to:

$$
\begin{aligned}
& 2 \pi \sum_{a=1}^{\infty} \sum_{\substack{r|\nu \\
r| a}} \epsilon_{p}(r) H_{a / r}\left(m, \frac{p \nu \nu^{\prime}}{r^{2}}\right) \sqrt{\frac{m}{\nu \nu^{\prime}}} I_{2 s-1}\left(\frac{4 \pi}{a} \sqrt{m p\left|\nu \nu^{\prime}\right|}\right) \mathcal{K}_{s}\left(2 \pi \nu y_{1}\right) \mathcal{K}_{s}\left(2 \pi \nu^{\prime} y_{2}\right) \\
& =2 \pi \sqrt{p} \sum_{r \mid \nu} \frac{\epsilon_{p}(r)}{r} \sqrt{\frac{m r^{2}}{p \nu \nu^{\prime}}} \sum_{c=1}^{\infty} H_{c}\left(m, \frac{p \nu \nu^{\prime}}{r^{2}}\right) I_{2 s-1}\left(\frac{4 \pi}{c r} \sqrt{m p\left|\nu \nu^{\prime}\right|}\right) \mathcal{K}_{s}\left(2 \pi \nu y_{1}\right) \mathcal{K}_{s}\left(2 \pi \nu^{\prime} y_{2}\right) .
\end{aligned}
$$

By Theorem 2.1] we finally find for the $\nu$-th coefficient:

$$
\sqrt{p} \sum_{r \mid \nu} \frac{\epsilon_{p}(r)}{r} b_{m}\left(p \nu \nu^{\prime} / r^{2}, s\right) \mathcal{K}_{s}\left(2 \pi \nu y_{1}\right) \mathcal{K}_{s}\left(2 \pi \nu^{\prime} y_{2}\right) .
$$


In the same way one can show that for $\nu \in \partial_{F}^{-1}$ with $\nu \nu^{\prime}<0$ the $\nu$-th coefficient of $\tilde{\Phi}_{m p^{2}}\left(z_{1}, z_{2}, s\right)-\tilde{\Phi}_{m p^{2}}^{0}\left(z_{1}, z_{2}, s\right)$ is equal to

$$
\begin{aligned}
& \sqrt{p} \sum_{r \mid \nu} \frac{\epsilon_{p}(r)}{r} b_{m}\left(p \nu \nu^{\prime} / r^{2}, s\right) \mathcal{K}_{s}\left(2 \pi \nu y_{1}\right) \mathcal{K}_{s}\left(2 \pi \nu^{\prime} y_{2}\right) \\
& +\sqrt{p} \sum_{r \mid \nu} \frac{\epsilon_{p}(r)}{r} \delta_{-m, p \nu \nu^{\prime} / r^{2}} \mathcal{K}_{s}\left(2 \pi \nu y_{1}\right) \mathcal{K}_{s}\left(2 \pi \nu^{\prime} y_{2}\right) .
\end{aligned}
$$

Here the extra contribution with the Kronecker delta comes from the Kronecker delta $\delta_{-m, n}$ in the formula for $b_{m}(n, s)$ with $n<0$.

If we put the above contributions together, and use in addition the formula for $\tilde{\Phi}_{m p^{2}}^{0}$ of Lemma 4.1, we find

$$
\begin{aligned}
& \tilde{\Phi}_{m p^{2}}\left(z_{1}, z_{2}, s\right) \\
& =\left(\frac{p}{\pi}\right)^{s-1 / 2} \Gamma(s-1 / 2) L\left(2 s-1, \epsilon_{p}\right) b_{m}(0, s)\left(y_{1} y_{2}\right)^{1-s} \\
& +\sqrt{p} \sum_{\substack{\nu \in \partial_{F}^{-1} \\
\nu \nu^{\prime} \neq 0}} \sum_{r \mid \nu} \frac{\epsilon_{p}(r)}{r} b_{m}\left(p \nu \nu^{\prime} / r^{2}, s\right) \mathcal{K}_{s}\left(2 \pi \nu y_{1}\right) \mathcal{K}_{s}\left(2 \pi \nu^{\prime} y_{2}\right) e\left(\nu x_{1}+\nu^{\prime} x_{2}\right) \\
& +\sqrt{p} \sum_{\substack{\nu \in \partial_{F}^{-1} \\
\nu \nu^{\prime}<0}} \sum_{r \mid \nu} \frac{\epsilon_{p}(r)}{r} \delta_{-m, p \nu \nu^{\prime} / r^{2}} \mathcal{K}_{s}\left(2 \pi \nu y_{1}\right) \mathcal{K}_{s}\left(2 \pi \nu^{\prime} y_{2}\right) e\left(\nu x_{1}+\nu^{\prime} x_{2}\right) \\
& +2 \sqrt{p} \sum_{\substack{\lambda \in \partial_{F}^{-1} \\
\mathrm{~N}(\lambda)=-m / p}} \sum_{n \geq 1} \frac{\epsilon_{p}(n)}{n} \mathcal{I}_{s}\left(2 \pi n \beta\left(\lambda y_{1}, \lambda^{\prime} y_{2}\right)\right) \mathcal{K}_{s}\left(2 \pi n \alpha\left(\lambda y_{1}, \lambda^{\prime} y_{2}\right)\right) e\left(n \lambda x_{1}+n \lambda^{\prime} x_{2}\right) .
\end{aligned}
$$

By rearranging the sums one deduces the stated formula.

Now it can be proved as in $[\mathrm{Br} 1]$ that $\tilde{\Phi}_{m p^{2}}\left(z_{1}, z_{2}, s\right)$ has a meromorphic continuation in $s$ to a neighborhood of $s=1$. The continuation turns out to be holomorphic at $s=1$, whereas there always was a simple pole in [Br1]. This follows from the presence of $L\left(2 s-1, \epsilon_{p}\right)$ in the constant term of the Fourier expansion given in Theorem 4.4, while there appeared $\zeta(2 s-1)$ in Br1] Theorem 1.

Definition 4.5. We define the regularized Green function $\tilde{\Phi}_{m p^{2}}\left(z_{1}, z_{2}\right)$ for $\tilde{T}_{m}$ as the value of $\tilde{\Phi}_{m p^{2}}\left(z_{1}, z_{2}, s\right)$ at $s=1$.

One finds that $\tilde{\Phi}_{m p^{2}}\left(z_{1}, z_{2}\right)$ is a harmonic function on $\mathbb{H}^{2} \backslash \tilde{T}_{m}$ with a logarithmic singularity along $-2 \tilde{T}_{m}$. 
TWISTED BORCHERDS PRODUCTS ON HILBERT MODULAR SURFACES

Theorem 4.6. The Green function $\tilde{\Phi}_{m p^{2}}\left(z_{1}, z_{2}\right)$ associated to $\tilde{T}_{m}$ has the Fourier expansion

$$
\begin{aligned}
& \tilde{\Phi}_{m p^{2}}\left(z_{1}, z_{2}\right) \\
& =\sqrt{p} L\left(1, \epsilon_{p}\right) b_{m}(0,1) \\
& +\sqrt{p} \sum_{\substack{\nu \in \partial_{F}^{-1} \\
\nu \nu^{\prime}>0}} \sum_{n \geq 1} \frac{\epsilon_{p}(n)}{n} b_{m}\left(p \nu \nu^{\prime}, 1\right) e\left(-2 \pi n\left|\nu y_{1}+\nu^{\prime} y_{2}\right|\right) e\left(\nu n x_{1}+\nu^{\prime} n x_{2}\right) \\
& +\sqrt{p} \sum_{\substack{\lambda \in \partial_{F}^{-1} \\
\mathrm{~N}(\lambda)=-m / p}} \sum_{n \geq 1} \frac{\epsilon_{p}(n)}{n} e\left(-2 \pi n\left|\lambda y_{1}+\lambda^{\prime} y_{2}\right|\right) e\left(n \lambda x_{1}+n \lambda^{\prime} x_{2}\right) .
\end{aligned}
$$

It converges normally on $y_{1} y_{2}>m p$ outside the polar part of $\tilde{T}_{m}$.

Proof. This follows immediately from Theorem 4.4 (2.3), and (2.4), noting that $b_{m}(n, 1)=$ 0 when $n<0$, and that $\alpha\left(r_{1}, r_{2}\right)-\beta\left(r_{1}, r_{2}\right)=\left|r_{1}+r_{2}\right|$ for $r_{1}, r_{2} \in \mathbb{R}$ with $r_{1} r_{2}<0$.

Remark 4.7. We have $\sqrt{p} L\left(1, \epsilon_{p}\right)=h_{F} \log \left(\varepsilon_{0}\right)$, where $h_{F}$ denotes the class number of $F$ and $\varepsilon_{0}>1$ the fundamental unit.

\section{Twisted Borcherds PROducts}

As an application of Theorem [4.6] of the previous section we obtain a variant of the Borcherds lift for Hilbert modular surfaces (see [Bo2, [Br1], BB]). It can be viewed as a multiplicative analogue of the Doi-Naganuma lift [DN] from holomorphic modular forms of weight $k$ for $\Gamma^{\prime}$ to Hilbert modular forms of weight $k$ for $\Gamma$. Its existence was suggested by Zagier in Za3].

Following Za3] §7, we define

$$
R_{p}(t)=\prod_{b(p)}(1-e(b / p) t)^{\epsilon_{p}(b)} .
$$

It is a rational function of $t$ with coefficients in $F$.

Lemma 5.1. The function $R_{p}(t) \in F(t)$ has the following properties:

(i) $R_{p}(t)^{\prime}=R_{p}(t)^{-1}$.

(ii) $R_{p}\left(t^{-1}\right)=R_{p}(t)$.

(iii) $\log \left(R_{p}(t)\right)=-\sqrt{p} \sum_{n \geq 1} \frac{1}{n} \epsilon_{p}(n) t^{n}$.

Proof. The first two properties are verified by direct computation. The third property follows from the identity $\sum_{b(p)} \epsilon_{p}(b) e(b n / p)=\sqrt{p} \epsilon_{p}(n)$.

Theorem 5.2. Let $f=\sum_{n \gg-\infty} c(n) q^{n} \in \mathbb{Z}[j]$ be a weakly holomorphic modular form of weight 0 for $\Gamma^{\prime}$ with integral Fourier coefficients. Then there exists a symmetric meromorphic Hilbert modular function $\Psi(z, f)$ for $\Gamma$ (of weight 0 , with trivial multiplier system, defined over F) such that: 
(i) The divisor of $\Psi(z, f)$ is given by

$$
\operatorname{div}(\Psi(z, f))=\sum_{n>0} c(-n) \tilde{T}_{n}
$$

(ii) The function $\Psi(z, f)$ has the Borcherds product expansion

$$
\begin{aligned}
\Psi(z, f) & =\prod_{\substack{\nu \in \partial_{F}^{-1} \\
\nu>0}} \prod_{b(p)}\left(1-e\left(\frac{b}{p}+\nu z_{1}+\nu^{\prime} z_{2}\right)\right)^{\epsilon_{p}(b) c\left(p \nu \nu^{\prime}\right)} \\
& =\prod_{\substack{\nu \in \partial_{F}^{-1} \\
\nu>0}} R_{p}\left(e\left(\nu z_{1}+\nu^{\prime} z_{2}\right)\right)^{c\left(p \nu \nu^{\prime}\right)},
\end{aligned}
$$

which converges normally for all $\left(z_{1}, z_{2}\right)$ with $y_{1} y_{2}>N p$ outside the set of poles, where $N=\max \{n \in \mathbb{Z} ; c(-n) \neq 0\}$.

(iii) The lifting is multiplicative, i.e., if $f, g \in \mathbb{Z}[j]$, then $\Psi(f+g)=\Psi(f) \Psi(g)$.

(iv) We have

$$
\log |\Psi(z, f)|=-\frac{1}{2} \sum_{n>0} c(-n)\left(\tilde{\Phi}_{n p^{2}}(z)-\sqrt{p} L\left(1, \epsilon_{p}\right) b_{n}(0,1)\right) .
$$

Proof. Let us first assume that $f=q^{-m}+O(1)$, as $q \rightarrow 0$, for some positive integer $m$. Then, according to Theorem 2.3. $f(\tau)=F_{m}(\tau, 1)+C$ for some constant $C \in \mathbb{Z}$. We define the function $\Psi(z, f)$ by the product expansion in (ii). In view of Theorem 4.6 and Lemma 5.1 (ii), (iii) we have

$$
\log |\Psi(z, f)|=-\frac{1}{2}\left(\tilde{\Phi}_{m p^{2}}(z)-\sqrt{p} L\left(1, \epsilon_{p}\right) b_{m}(0,1)\right) .
$$

In particular, the normal convergence of the Fourier expansion of $\tilde{\Phi}_{m p^{2}}(z)$ on $y_{1} y_{2}>m p$ implies the normal convergence of the infinite product $\Psi(z, f)$. Therefore (ii) and (iv) hold.

In the same way as in [Br1], Theorem 4, one can show that $\Psi(z, f)$ has a meromorphic continuation to all of $\mathbb{H}^{2}$ and that $\operatorname{div}(\Psi(z, f))=\tilde{T}_{m}$ : This follows from (5.2) and the fact that $\tilde{\Phi}_{m p^{2}}(z)$ is a pluriharmonic function on $\mathbb{H}^{2} \backslash \tilde{T}_{m}$ with a logarithmic singularity along the divisor $-2 \tilde{T}_{m}$.

Moreover, (5.2) implies that $|\Psi(z, f)|$ is invariant under the group $\Gamma$. The matrix $S=$ $\left(\begin{array}{cc}0 & -1 \\ 1 & 0\end{array}\right) \in \Gamma$ satisfies the relation $S^{2}=-1$. Consequently,

$$
\Psi(S z, f)= \pm \Psi(z, f) .
$$

On the other hand, it follows from the product expansion that $\Psi(z, f)$ is invariant under translations $\left(\begin{array}{ll}1 & \mu \\ 0 & 1\end{array}\right)$ where $\mu \in \mathcal{O}_{K}$. Using the relation $\left(S\left(\begin{array}{ll}1 & 1 \\ 0 & 1\end{array}\right)\right)^{3}=-1$ in $\Gamma$, we may conclude that the sign in (5.3) must be +1 . But, by a theorem of Vaserstein, the translations $\left(\begin{array}{ll}1 & \mu \\ 0 & 1\end{array}\right)$ and the matrix $S=\left(\begin{array}{cc}0 & -1 \\ 1 & 0\end{array}\right)$ generate the group $\Gamma$. Hence $\Psi(z, f)$ is invariant under $\Gamma$.

Moreover, the product expansion implies that $\Psi(z, f)$ can be written as the quotient of two holomorphic Hilbert modular forms with Fourier coefficients in $F$. Hence, by the $q$-expansion principle, $\Psi(z, f)$ is defined over $F$. 
Finally, the multiplicativity (iii) also follows from the infinite product expansion.

The condition $\nu>0$ under the product replaces the Weyl-chamber condition occurring for the untwisted Borcherds products $[\mathrm{BB}$. It has this easy form because of the presence of the character $\epsilon_{p}(b)$ which causes some cancellations. Because of Lemma 5.1 (ii) the product is actually over $\mathcal{O}_{F}^{\times} /\{ \pm 1\}$.

For the rest of this paper we write $\tilde{\Psi}_{m}\left(m \in \mathbb{Z}_{>0}\right)$ for the twisted Borcherds lift in the sense of Theorem 5.2 of the unique weakly holomorphic modular form

$$
J_{m}=q^{-m}+O(q) \in \mathbb{Z}[j] .
$$

Remark 5.3. In view of Lemma 5.1 (i), the conjugation in $F$ maps $\Psi(z, f)$ to $\Psi(z, f)^{-1}$.

\section{Class Fields}

Let $F=\mathbb{Q}(\sqrt{p})$ be a real quadratic field as before. Let $K=F(\sqrt{\Delta})$ be a non-biquadratic totally imaginary quadratic extension of $F$. We view both $K$ and $F\left(\sqrt{\Delta^{\prime}}\right)$ as subfields of $\mathbb{C}$ with $\sqrt{\Delta}, \sqrt{\Delta^{\prime}} \in \mathbb{H}$. Then $M=F\left(\sqrt{\Delta}, \sqrt{\Delta^{\prime}}\right)$ is Galois over $\mathbb{Q}$ and has an automorphism $\sigma$ of order 4 such that $\sigma(\sqrt{\Delta})=\sqrt{\Delta^{\prime}}$ and $\sigma\left(\sqrt{\Delta^{\prime}}\right)=-\sqrt{\Delta}$. The field $K$ has four CM types: $\Phi=\{1, \sigma\}, \sigma \Phi=\left\{\sigma, \sigma^{2}\right\}, \sigma^{2} \Phi$, and $\sigma^{3} \Phi$. We assume that the relative discriminant $d_{K / F}$ of $K / F$ satisfies the technical condition

$$
d_{K / F} \cap \mathbb{Z}=q \mathbb{Z}, \quad \mathrm{N}_{F / \mathbb{Q}} d_{K / F}=q,
$$

for a prime number $q \equiv 1(\bmod 4)$.

Recall that the Hilbert modular surface $X$ corresponding to $\Gamma=\mathrm{SL}_{2}\left(\mathcal{O}_{F}\right)$ parameterizes isomorphism classes of triples $(A, \imath, m)$, where $(A, \imath)$ is an abelian surface with real multiplication $\imath: \mathcal{O}_{F} \hookrightarrow \operatorname{End}(A)$, and

$$
m:\left(\mathfrak{M}_{A}, \mathfrak{M}_{A}^{+}\right) \longrightarrow\left(\partial_{F}^{-1}, \partial_{F}^{-1,+}\right)
$$

is an $\mathcal{O}_{F}$-isomorphism between the polarization module $\mathfrak{M}_{A}$ of $A$ and $\partial_{F}^{-1}$, taking the subset of polarizations to totally positive elements of $\partial_{F}^{-1}$ (see e.g. Go, Theorem 2.17 and BY] Section 3).

Let $\mathcal{C} \mathcal{M}\left(K, \Phi, \mathcal{O}_{F}\right)$ be the CM 0 -cycle in $X$ of CM abelian surfaces of CM type $(K, \Phi)$, i.e., the points on $X$ with an $\mathcal{O}_{K}$-action via $\Phi$ (see [BY] Section 3 for details). The field of moduli for the CM cycle $\mathcal{C} \mathcal{M}(K):=\mathcal{C} \mathcal{M}\left(K, \Phi, \mathcal{O}_{F}\right)+\mathcal{C} \mathcal{M}\left(K, \sigma^{3} \Phi, \mathcal{O}_{F}\right)$ is $\mathbb{Q}$.

Let $(\tilde{K}, \tilde{\Phi})$ be the reflex of $(K, \Phi)$ with maximal totally real subfield $\tilde{F}=\mathbb{Q}\left(\sqrt{\Delta^{\prime}}\right)$. Let $I(K)$ be the group of all fractional ideals of $K$, and let $H(K)$ be the subgroup of $I(K)$ of ideals $\mathfrak{a}$ such that

$$
N_{\Phi} \mathfrak{a}=\mu \mathcal{O}_{\tilde{K}}, \quad N \mathfrak{a}=\mu \bar{\mu} \quad \text { for some } \mu \in \tilde{K}^{*} .
$$

Here $N \mathfrak{a}=\# \mathcal{O}_{K} / \mathfrak{a}$, and $N_{\Phi}$ is the type norm from $I(K)$ to $I(\tilde{K})$ given by, in this case,

$$
N_{\Phi}(\mathfrak{a})=N_{M / \tilde{K}}\left(\mathfrak{a} \mathcal{O}_{M}\right) .
$$

We call the quotient $\mathcal{C C}(K)=\mathcal{C C}(K, \Phi)=I(K) / H(K)$ the CM ideal class group of $K$. According to [Sh], page 112, Main Theorem 1, the class field $H_{\tilde{K}}$ of $\tilde{K}$ associated to the 
CM ideal class group $\mathcal{C C}(\tilde{K}, \tilde{\Phi})$ is the composite of $\tilde{K}$ with the field of the moduli of any polarized $\mathrm{CM}$ abelian variety of type $(K, \Phi)$ by $\mathcal{O}_{K}$. Recall that $\mathcal{C C}(\tilde{K}, \tilde{\Phi})=\operatorname{Gal}\left(H_{\tilde{K}} / \tilde{K}\right)$ acts on $\mathcal{C} \mathcal{M}\left(K, \Phi, \mathcal{O}_{F}\right)$ via $[\mathrm{BY}],(3.7)$.

Inspired by the classical result that $j\left(\frac{-D+\sqrt{-D}}{2}\right)$ generates the Hilbert class field of the imaginary quadratic field $\mathbb{Q}(\sqrt{-D})$, we consider whether $H_{\tilde{K}}$ has a 'canonical' generator over $\tilde{K}$. Since $F$ is in general not contained in $\tilde{K}$, it seems more natural to consider the field $L_{K}=K H_{\tilde{K}}=M H_{\tilde{K}}=F H_{\tilde{K}}$ over either $M$ or $F$, where $M=K \tilde{K}$ is the smallest Galois extension of $\mathbb{Q}$ containing $K$ and/or $\tilde{K}$. For example, a natural question is whether $L_{K}=M\left(\tilde{\Psi}_{m}(z)\right)$ for some $m$ and some $\mathrm{CM}$ point $z \in \mathcal{C} \mathcal{M}\left(K, \Phi, \mathcal{O}_{F}\right)$ ?

Lemma 6.1. Assume that $d_{K}=p^{2} q$ with $p \equiv q \equiv 1 \bmod 4$ being odd primes. Then $\mathcal{C C}(\tilde{K}, \tilde{\Phi})$ acts on $\mathcal{C} \mathcal{M}\left(K, \Phi, \mathcal{O}_{F}\right)$ simply transitively.

Proof. Let $\mathrm{CL}_{0}(K)$ be the subgroup of $\mathrm{CL}(K)$ generated by ideal classes $[\mathfrak{a}]$ such that

$$
N_{K / F} \mathfrak{a}=\mu \mathcal{O}_{F}, \quad \mu \gg 0 \text { (totally positive). }
$$

Then [BY] Lemma 5.3 asserts that the type norm $N_{\tilde{\Phi}}$ gives an isomorphism between $\mathrm{CL}_{0}(\tilde{K})$ and $\mathrm{CL}_{0}(K)$. It is clear by definition that $N_{\tilde{\Phi}}$ maps $I(\tilde{K})$ to $\mathrm{CL}_{0}(K)$, so it is surjective. It is easy to check that the kernel is exactly $H(\tilde{K})$. Indeed, if $[\mathfrak{a}] \in \operatorname{ker} N_{\tilde{\Phi}}$, then

$$
N_{\tilde{\Phi}} \mathfrak{a}=\mu \mathcal{O}_{K} .
$$

This implies

$$
N \mathfrak{a} \mathcal{O}_{F}=N_{K / F} N_{\tilde{\Phi}} \mathfrak{a}=\mu \bar{\mu} \mathcal{O}_{F}
$$

and thus

$$
N \mathfrak{a}=\mu \bar{\mu} \epsilon
$$

for some unit $\epsilon$. Clearly $\epsilon$ is totally positive and thus a square $\epsilon_{1}^{2}$ since $F=\mathbb{Q}(\sqrt{p})$. Replacing $\mu$ by $\mu \epsilon_{1}$, one sees that $\mathfrak{a} \in H(\tilde{K})$. So

$$
\mathcal{C C}(\tilde{K}) \cong \mathrm{CL}_{0}(K)
$$

and $\left[L_{K}: M\right]=\left[H_{\tilde{K}}: \tilde{K}\right]=\# \mathrm{CL}_{0}(K)$. On the other hand, according to the remark after BY], Lemma 3.3, The forgetful map

$$
\mathcal{C} \mathcal{M}\left(K, \Phi, \mathcal{O}_{F}\right) \rightarrow \mathrm{CL}_{0}(K), \quad\left(\mathbb{C}^{2} / \Phi(\mathfrak{a}), \imath, m\right) \rightarrow \mathfrak{a}
$$

is a bijection. So $\mathcal{C C}(\tilde{K})$ acts on $\mathcal{C} \mathcal{M}\left(K, \Phi, \mathcal{O}_{F}\right)$ simply transitively.

Corollary 6.2. Let the assumption be as in Lemma 6.1] Let $z=(A, \imath, m) \in \mathcal{C} \mathcal{M}\left(K, \Phi, \mathcal{O}_{F}\right)$ and let $k_{z}$ be the field of definition of $z$ containing $\tilde{F}$. Then

$$
\tilde{\Psi}_{m}\left(\mathcal{C} \mathcal{M}\left(K, \Phi, \mathcal{O}_{F}\right)\right)=N_{M_{z} / M^{+}} \tilde{\Psi}_{m}(z) .
$$

Here $M_{z}=F k_{z}=M^{+} k_{z}$, and $M^{+}=F \tilde{F}$ is the maximal totally real subfield of $M$. 
Proof. Recall that $k_{z}$ is the field of moduli of $(A, \imath, m)$ and that $H_{\tilde{K}}=\tilde{K} k_{z}$. Lemma 3.4(1) of [BY] asserts that $\mathcal{C} \mathcal{M}\left(K, \Phi, \mathcal{O}_{F}\right)$ is defined over $\tilde{F}$. So the lemma above implies that $k_{z}$ does not contain $\tilde{K}$ and $\left[k_{z}: \tilde{F}\right]=\# \mathcal{C C}(\tilde{K})$, and different embeddings of $k_{z}$ into $\mathbb{C}$ fixing $\tilde{F}$ map z into different $\mathrm{CM}$ points in $\mathcal{C} \mathcal{M}\left(K, \Phi, \mathcal{O}_{F}\right)$. Since $\tilde{\Psi}_{m}$ is defined over $F$, we obtain the corollary.

Corollary 6.3. Let the assumption be as in Lemma 6.1. If $\tilde{\Psi}_{m}(\mathcal{C M}(K))$ is not a k-power in $F$ for any odd integer $k>1$, then $\tilde{\Psi}_{m}(z)$ generates $L_{K}$ over $M$.

Proof. Recall that $\mathcal{C} \mathcal{M}(K)=\mathcal{C} \mathcal{M}\left(K, \Phi, \mathcal{O}_{F}\right)+\mathcal{C} \mathcal{M}\left(K, \sigma^{3} \Phi, \mathcal{O}_{F}\right)$. If $\tilde{\Psi}_{m}(z)$ does not generate $L_{K}$ over $M$, then it does not generate $M^{+} k_{z}$ over $M^{+}$. Let $L$ be the subfield of $M^{+} k_{z}$ generated by $\tilde{\Psi}_{m}(z)$ over $M^{+}$. Then the above corollary implies that

$$
\tilde{\Psi}_{m}\left(\mathcal{C M}\left(K, \Phi, \mathcal{O}_{F}\right)\right)=\left(N_{L / M^{+}}\left(\tilde{\Psi}_{m}(z)\right)\right)^{\left[M^{+} k_{z}: L\right]}
$$

is a $k$-th power with $k=\left[M^{+} k_{z}: L\right]>1$. Therefore

$$
\tilde{\Psi}_{m}(\mathcal{C M}(K))=N_{M^{+} / F} \tilde{\Psi}_{m}\left(\mathcal{C} \mathcal{M}\left(K, \Phi, \mathcal{O}_{F}\right)\right)=\left(N_{L / F}\left(\tilde{\Psi}_{m}(z)\right)\right)^{k} .
$$

Finally, the condition $d_{K}=p^{2} q$ implies that $d_{\tilde{K}}=q^{2} p$ is an odd number and thus its class number $h(\tilde{K})$ is odd [CH]. In particular, $k|\# \mathcal{C} \mathcal{C}(\tilde{K})| h(\tilde{K})$ is odd.

Remark 6.4. Let the notation be as above, and let $c=\tilde{\Psi}_{m}\left(\mathcal{C M}\left(K, \Phi, \mathcal{O}_{F}\right)\right) \in M^{+}$. The above proof implies

$$
N_{M^{+} / F} c=\tilde{\Psi}_{m}(\mathcal{C M}(K)) .
$$

On the other hand, it follows from Remark 5.3 that

$$
N_{M^{+} / \tilde{F}} c=1
$$

The numerical examples in Section [7] suggest that $\tilde{\Psi}_{m}(\mathcal{C M}(K))$ is a square and that $c \in F$, that is,

$$
\tilde{\Psi}_{m}\left(\mathcal{C M}\left(K, \Phi, \mathcal{O}_{F}\right)\right)=\tilde{\Psi}_{m}\left(\mathcal{C M}\left(K, \sigma^{3} \Phi, \mathcal{O}_{F}\right)\right)
$$

We will try to compute $\tilde{\Psi}_{m}(\mathcal{C} \mathcal{M}(K))$ in the next section.

Proposition 6.5. Assume that $d_{K}=p^{2} q$ with $p \equiv q \equiv 1 \bmod 4$ being odd primes, and let $B$ be a set of positive integers. Then $L_{K}=M\left(\tilde{\Psi}_{m}(z), m \in B\right)$ for a $z \in \mathcal{C} \mathcal{M}\left(K, \Phi, \mathcal{O}_{F}\right)$ if and only if the functions $\tilde{\Psi}_{m}(m \in B)$ separate the points in $\mathcal{C} \mathcal{M}\left(K, \Phi, \mathcal{O}_{F}\right)$.

Proof. First assume that the $\tilde{\Psi}_{m}(m \in B)$ separate the points in $\mathcal{C} \mathcal{M}\left(K, \Phi, \mathcal{O}_{F}\right)$. Clearly $L_{K} \supset M\left(\tilde{\Psi}_{m}(z), m \in B\right)$. Suppose that $\alpha \in \operatorname{Gal}\left(L_{K} / M\right)=\operatorname{Gal}\left(H_{\tilde{K}} / \tilde{K}\right)$ fixes the field $M\left(\tilde{\Psi}_{m}(z), m \in B\right)$. Then

$$
\tilde{\Psi}_{m}(\alpha(z))=\alpha\left(\tilde{\Psi}_{m}(z)\right)=\tilde{\Psi}_{m}(z)
$$

for every $m \in B$, and so $\alpha(z)=z$. This implies that $\alpha=1$, and $L_{K}=M\left(\tilde{\Psi}_{m}(z), m \in B\right)$. 
Conversely, if there are $z_{1} \neq z_{2} \in \mathcal{C} \mathcal{M}\left(K, \Phi, \mathcal{O}_{F}\right)$ such that $\tilde{\Psi}_{m}\left(z_{1}\right)=\tilde{\Psi}_{m}\left(z_{2}\right)$ for every $m \in B$, let $1 \neq \alpha \in \operatorname{Gal}\left(L_{K} / M\right)$ such that $\alpha\left(z_{1}\right)=z_{2}$, which exists by Lemma 6.1. Then

$$
\alpha\left(\tilde{\Psi}_{m}\left(z_{1}\right)\right)=\tilde{\Psi}_{m}\left(z_{2}\right)=\tilde{\Psi}_{m}\left(z_{1}\right)
$$

for every $m \in B$ and thus $M\left(\tilde{\Psi}_{m}(z), m \in B\right)$ is fixed by $\alpha$. So $L_{K} \neq M\left(\tilde{\Psi}_{m}(z), m \in B\right)$.

For two positive integers $m$ and $n$ such that $m n$ is not a square, it is known that $\tilde{T}_{m}$ and $\tilde{T}_{n}$ has no common component [HZ]. This implies that the rational map

$$
\phi_{m, n}: X \rightarrow \mathbb{P}^{1} \times \mathbb{P}^{1}, \quad z \mapsto\left(\tilde{\Psi}_{m}(z), \tilde{\Psi}_{n}(z)\right)
$$

is generically finite, i.e., $\left[\mathbb{C}(X): \mathbb{C}\left(\tilde{\Psi}_{m}(z), \tilde{\Psi}_{n}(z)\right)\right]$ is finite.

Theorem 6.6. Let $F=\mathbb{Q}(\sqrt{p})$ be a fixed real quadratic field. Let $K$ be a non-biquadratic $C M$ quartic field with maximal totally real subfield $F$ and discriminant $d_{K}=p^{2} q$ with $p \equiv q \equiv 1 \bmod 4$ primes. Let $z \in \mathcal{C} \mathcal{M}\left(K, \Phi, \mathcal{O}_{F}\right)$ and $L_{m, n}(K)=M\left(\tilde{\Psi}_{m}(z), \tilde{\Psi}_{n}(z)\right)$ be the unramified abelian extension of $M$ generated by $\tilde{\Psi}_{m}(z)$ and $\tilde{\Psi}_{n}(z)$. Then there are constants $c_{1}, c_{2}$ and $d>0$ depending on $F, m$, and $n$, but independent of $K$, such that

(i) $\left[L_{K}: L_{m, n}(K)\right] \leq d$.

(ii) $c_{1} \sqrt{q} \leq\left[L_{m, n}(K): M\right] \leq c_{2} \sqrt{q}$.

In particular $\lim _{q \rightarrow \infty}\left[L_{m, n}(K): M\right]=\infty$.

Proof. Lemma 6.1 implies that $\operatorname{Gal}\left(L_{K} / M\right)$ acts on $\mathcal{C} \mathcal{M}\left(K, \Phi, \mathcal{O}_{F}\right)$ simply transitively, so

$$
\mathcal{C M}\left(K, \Phi, \mathcal{O}_{F}\right)=\left\{\sigma(z) ; \sigma \in \operatorname{Gal}\left(L_{K} / M\right)\right\} .
$$

Here $z$ is a fixed $\mathrm{CM}$ point in $\mathcal{C} \mathcal{M}\left(K, \Phi, \mathcal{O}_{F}\right)$. Set

$$
\begin{aligned}
A & =\left\{z^{\prime} \in \mathcal{C} \mathcal{M}\left(K, \Phi, \mathcal{O}_{F}\right) ; \tilde{\Psi}_{i}\left(z^{\prime}\right)=\tilde{\Psi}_{i}(z), i=m, n\right\} \\
& =\left\{\sigma(z) ; \sigma \in \operatorname{Gal}\left(L_{K} / M\right), \tilde{\Psi}_{i}(\sigma(z))=\tilde{\Psi}_{i}(z), i=m, n\right\} .
\end{aligned}
$$

Since

$$
\sigma\left(\tilde{\Psi}_{m}(z)\right)=\tilde{\Psi}_{m}(\sigma(z))
$$

one has that $\sigma(z) \in A$ if and only is $\sigma \in \operatorname{Gal}\left(L_{K} / L_{m, n}(K)\right)$. So

$$
\left[L_{K}: L_{m, n}(K)\right]=\# A \leq \operatorname{deg} \phi_{m, n}
$$

generically, that is, if $\phi_{m, n}(z) \notin B=\left\{(a, b) ; \phi_{m, n}^{-1}(a, b)\right.$ is infinite $\}$. Notice that $B$ is a finite set. Since $\phi_{m, n}$ is defined over $F, \phi_{m, n}(z) \in B$ for some $z \in \mathcal{C} \mathcal{M}\left(K, \Phi, \mathcal{O}_{F}\right)$ implies that $\phi_{m, n}(\sigma(z)) \in B$ for all $\sigma(z) \in \mathcal{C} \mathcal{M}\left(K, \Phi, \mathcal{O}_{F}\right)$, i.e.,

$$
\mathcal{C M}\left(K, \Phi, \mathcal{O}_{F}\right) \subset \phi_{m, n}^{-1}(B) \quad \text { or } \quad \mathcal{C} \mathcal{M}\left(K, \Phi, \mathcal{O}_{F}\right) \cap \phi_{m, n}^{-1}(B)=\emptyset .
$$

According to the equidistribution theorem of CM points on a Hilbert modular variety recently proved by Zhang (cf. [Zh], Theorem 2.1), $\left\{\mathcal{C} \mathcal{M}\left(K, \Phi, \mathcal{O}_{F}\right) ; d_{K}=p^{2} q\right\}$ is equidistributed on $X$. On the other hand, $\phi_{m, n}^{-1}(B)$ is just a divisor of $X$, so there is $q_{0}>0$ such that $q>q_{0}$ implies that $\mathcal{C M}\left(K, \Phi, \mathcal{O}_{F}\right) \cap \phi_{m, n}^{-1}(B)$ is empty. Let

$$
d_{0}=\max \left\{\left[L_{K}: L_{m, n}(K)\right] ; q \leq q_{0}\right\}, \quad \text { and } \quad d=\max \left\{\operatorname{deg} \phi_{m, n}, d_{0}\right\} .
$$


Then we always find

$$
\left[L_{K}: L_{m, n}(K)\right] \leq d
$$

This proves (i).

For (ii), one has

$$
\left[L_{K}: M\right]=\# \mathcal{C} \mathcal{M}\left(K, \Phi, \mathcal{O}_{F}\right)=\# \mathrm{CL}_{0}(K)=c \frac{h_{K} R_{K}}{h_{F} R_{F}} .
$$

Here $h_{F}$ and $h_{K}$ are the class numbers of $F$ and $K$ respectively, $R_{F}$ and $R_{K}$ are the regulators of $F$ and $K$ respectively, and $\frac{1}{2} \leq c \leq 2$. Indeed,

$$
\frac{R_{F}}{R_{K}} \leq c=\left[\mathrm{CL}(F): N_{K / F} \mathrm{CL}(K)\right] \frac{R_{F}}{R_{K}} \leq\left[\mathrm{CL}(F): N_{K / F} \mathrm{CL}(K)\right] .
$$

Since $F$ is fixed, one sees from the Brauer-Siegel theorem (cf. [La], page 321) that

$$
\left[L_{K}: M\right] \sim \sqrt{q}
$$

Now (ii) follows from (i).

Proposition 6.7. The field $L_{K}$ is Galois over F.

Proof. By Sh], page 112, Main Theorem 1, $H_{\tilde{K}}$ is the field of moduli of a CM abelian variety $z=(A, \imath, m) \in \mathcal{C} \mathcal{M}\left(K, \Phi, \mathcal{O}_{F}\right)$ over $\tilde{K}$. So $L_{K}$ is the field of moduli of a $\mathrm{CM}$ abelian variety $z \in \mathcal{C} \mathcal{M}\left(K, \Phi, \mathcal{O}_{F}\right)$ over $M$. Let $\alpha$ be an embedding of $L_{K}$ into $\bar{F}$ fixing $F$, and $\left.\alpha\right|_{K}$ is either the identity or the complex conjugation. So $\left(A^{\alpha}, i^{\alpha}, m^{\alpha}\right) \in \mathcal{C} \mathcal{M}\left(K, \Phi, \mathcal{O}_{F}\right)$ or $\mathcal{C} \mathcal{M}\left(K, \bar{\Phi}, \mathcal{O}_{F}\right)$. Clearly, $\left(K, \Phi, \mathcal{O}_{F}\right)$ and $\left(K, \bar{\Phi}, \mathcal{O}_{F}\right)$ have the same reflex field $\tilde{K}$, and the associated CM ideal class groups of $\tilde{K}$ are the same. This implies $\alpha\left(L_{K}\right)=L_{K}$, i.e., $L_{K}$ is Galois over $F$.

In general, $L_{K}$ is not abelian over $F$. Let $L_{F}$ be the composite of all $L_{K}$ with $K$ running through non-biquadratic CM quadratic extensions of $F$. Then one has an exact sequence

$$
1 \rightarrow \mathcal{A} \rightarrow \operatorname{Gal}\left(L_{F} / F\right) \rightarrow(\mathbb{Z} / 2)^{(\mathbb{N})} \rightarrow 0
$$

for some abelian group $\mathcal{A}$. It might be interesting to study the Galois group $\operatorname{Gal}\left(L_{F} / F\right)$. We end this section with the following question.

Question 6.8. Is $L_{K}$ independent of the choice of the CM types of $K$ ? This is equivalent to the question whether $L_{K}$ is Galois over $\mathbb{Q}$.

\section{EXAMPLES}

It would be very interesting to obtain closed formulas for the values of twisted Borcherds products at the CM cycles considered in the previous section in analogy to [BY]. However, since the twisted Borcherds products are in general only defined over $F$ (in contrast to the untwisted Borcherds products, which are essentially defined over $\mathbb{Q}$ ), their CM values will lie in $F$. This makes computations more difficult. Moreover, taking the norm to $\mathbb{Q}$ does not provide any insight, since $\Psi(z, f) \cdot \Psi(z, f)^{\prime}=1$ because of Remark 5.3. Note that for numeric computations, in the same way as in [BY], the problem arises that the product expansion of Theorem 5.2 only converges near the cusps. The CM points usually do not 
lie in the domain of convergence. Therefore one has to find an alternative expression for the twisted Borcherds products one wants to evaluate.

Here we discuss some examples in the special case $p=5$ where $F=\mathbb{Q}(\sqrt{5})$. The fundamental unit of $\mathcal{O}_{F}$ is equal to $\omega=\frac{1+\sqrt{5}}{2}$. The structure of the graded ring of holomorphic Hilbert modular forms for the group $\Gamma=\mathrm{SL}_{2}\left(\mathcal{O}_{F}\right)$ was determined by Gundlach [Gu], see also $\mathrm{Mü}$. In particular, it turns out that the graded ring $M_{2 *}^{\text {sym }}(\Gamma)$ of holomorphic symmetric Hilbert modular forms of even weight for $\Gamma$ is the polynomial ring $\mathbb{C}\left[g_{2}, g_{6}, g_{10}\right]$, where $g_{k}$ denotes the Eisenstein series (in the cusp $\infty$ for $\Gamma$ ) of weight $k$ normalized such that the constant term is 1 . Often it is more convenient to replace the generators $g_{6}$ and $g_{10}$ by the cusp forms

$$
\begin{aligned}
s_{6} & =67 \cdot\left(2^{5} \cdot 3^{3} \cdot 5^{2}\right)^{-1} \cdot\left(g_{2}^{3}-g_{6}\right), \\
s_{10} & =\left(2^{10} \cdot 3^{5} \cdot 5^{5} \cdot 7\right)^{-1} \cdot\left(2^{2} \cdot 3 \cdot 7 \cdot 4231 \cdot g_{2}^{5}-5 \cdot 67 \cdot 2293 \cdot g_{2}^{2} \cdot g_{6}+412751 \cdot g_{10}\right) .
\end{aligned}
$$

We have

$$
M_{2 *}^{\text {sym }}(\Gamma)=\mathbb{C}\left[g_{2}, s_{6}, s_{10}\right]
$$

Notice that $g_{2}, s_{6}, s_{10}$ all have rational integral and coprime Fourier coefficients. The cusp form $s_{10}$ is equal to the (untwisted) Borcherds lift $\Psi_{1}^{2}$ in the sense of [BB].

Recall from Section [5 that $\tilde{\Psi}_{m}$ is the symmetric Hilbert modular function of weight 0 which is the twisted Borcherds lift in the sense of Theorem 5.2 of the unique weakly holomorphic modular form $J_{m}=q^{-m}+O(q) \in \mathbb{Z}[j]$. By Gundlach's theorem, $\tilde{\Psi}_{m}$ must be a rational function in $g_{2}, s_{6}, s_{10}$ with coefficients in $F$. We now discuss how it can be computed.

For simplicity, we assume that $m$ is a square-free positive integer. Then the HirzebruchZagier divisor $T_{m p^{2}}$ decomposes into irreducible components $T_{m p^{2}}=T_{m}+F_{m p^{2}}^{+}+F_{m p^{2}}^{-}$. Recall that $\operatorname{div}\left(\tilde{\Psi}_{m}\right)=F_{m p^{2}}^{+}-F_{m p^{2}}^{-}$. On the other hand we can construct a symmetric holomorphic Hilbert modular form with divisor $F_{m p^{2}}^{+}+F_{m p^{2}}^{-}$of weight $k_{m}>0$ by taking the (untwisted) Borcherds lift $H_{m}=\Psi_{m p^{2}} / \Psi_{m}$ in the sense of [BB] Theorem 9. For instance, for $m=1$ we have $k_{1}=60$, and $H_{1}$ it is obtained as the lift of the unique weakly holomorphic modular form $h \in W_{0}^{+}\left(p, \epsilon_{p}\right)$ whose Fourier expansion has the form

$$
h=\frac{1}{2} q^{-25}-q^{-1}+60+438864 q+45271325304 q^{4}+\ldots
$$

The product $\tilde{\Psi}_{m} \cdot H_{m}$ is also a symmetric holomorphic Hilbert modular form of weight $k_{m}$. Its divisor is equal to $2 F_{m p^{2}}^{+}$. Hence there exist holomorphic Hilbert modular forms $\Psi_{m}^{+}$ and $\Psi_{m}^{-}$of weight $k_{m} / 2$ for $\Gamma$ such that $\left(\Psi_{m}^{+}\right)^{2}=H_{m} \cdot \tilde{\Psi}_{m}$ and $\left(\Psi_{m}^{-}\right)^{2}=H_{m} / \tilde{\Psi}_{m}$.

The function $\Psi_{m}^{ \pm}$must also be symmetric, because any skew-symmetric Hilbert modular form automatically vanishes on $F_{1}$, contradicting $\operatorname{div}\left(\Psi_{m}^{ \pm}\right)=F_{m p^{2}}^{ \pm}$. Moreover, $\Psi_{m}^{ \pm}$is defined 
over $F$ and

$$
\begin{aligned}
\left(\Psi_{m}^{+}\right)^{\prime} & =\Psi_{m}^{-}, \\
\tilde{\Psi}_{m} & =\Psi_{m}^{+} / \Psi_{m}^{-}, \\
H_{m} & =\Psi_{m}^{+} \cdot \Psi_{m}^{-}
\end{aligned}
$$

By Gundlach's theorem, $\Psi_{m}^{ \pm}$is a homogeneous polynomial in $g_{2}, s_{6}, s_{10}$ with coefficients in $F$. Using the infinite product expansions, this polynomial can be determined explicitly. Its degree depends on the weight $k_{m}$. Unfortunately, it turns out that the $k_{m}$ are rather large which makes computations difficult. The smallest weights that occur are $k_{1}=k_{2}=60$. For all $m$ the weight $k_{m}$ is divisible by 60 , and if $m>2$ then $k_{m} \geq 120$.

A computation with Maple shows that

$$
\begin{aligned}
16 \Psi_{1}^{+}= & (6+2 \sqrt{5}) g_{2}^{10} s_{10}+(10-2 \sqrt{5}) g_{2}^{9} s_{6}^{2}+(-2308750-1031750 \sqrt{5}) g_{2}^{7} s_{10} s_{6} \\
& +(-1220450-543450 \sqrt{5}) g_{2}^{6} s_{6}^{3}+(856853809375+383196837500 \sqrt{5}) g_{2}^{5} s_{10}^{2} \\
& +(-133751887500-59814018750 \sqrt{5}) g_{2}^{4} s_{10} s_{6}^{2} \\
& +(-309550426875-138434703750 \sqrt{5}) g_{2}^{3} s_{6}^{4} \\
& +(23003309053125000+10287392475000000 \sqrt{5}) g_{2}^{2} s_{10}^{2} s_{6} \\
& +(-18093694595625000-8091745695000000 \sqrt{5}) g_{2} s_{10} s_{6}^{3} \\
& +(-16048066250700000-7176913583400000 \sqrt{5}) s_{6}^{5} \\
& +(24527175191718750000+10968886216250000000 \sqrt{5}) s_{10}^{3} .
\end{aligned}
$$

Moreover,

$$
\begin{aligned}
\Psi_{2}^{+}= & -g_{2}^{15}+(48072800+21493760 \sqrt{5}) g_{2}^{12} s_{6} \\
& +(-12166677513088000-5441103582617600 \sqrt{5}) g_{2}^{10} s_{10} \\
& +(4809336551424000 \sqrt{5}+10754003449472000) g_{2}^{9} s_{6}^{2} \\
& +(-343213552017810432000000-153489766622216192000000 \sqrt{5}) g_{2}^{7} s_{10} s_{6} \\
& +(356570717554737254400000+159463272647655096320000 \sqrt{5}) g_{2}^{6} s_{6}^{3} \\
& +(-1295691015382296818073600000000-579450637646108270264320000000 \sqrt{5}) g_{2}^{5} s_{10}^{2} \\
& +(773852433537584819077120000000 \sqrt{5}+1730386645943677691904000000000) g_{2}^{4} s_{10} s_{6}^{2} \\
& +(-463842956129634468495360000000-207436876158063085617152000000 \sqrt{5}) g_{2}^{3} s_{6}^{4} \\
& +(26896303882903769962250240000000000 \sqrt{5}+60141963825664373337292800000000000) g_{2}^{2} s_{10}^{2} s_{6} \\
& +(-89933914258012151985733632000000000 \sqrt{5}-201098345763552732433612800000000000) g_{2} s_{10} s_{6}^{3} \\
& +(66189203273169170168775966720000000 \sqrt{5}+148003557895357846527777177600000000) s_{6}^{5} \\
& +(-17950573674763054301052928000000000000 \sqrt{5}-40138702971888390552944640000000000000) s_{10}^{3} .
\end{aligned}
$$

These polynomial representations can be used to calculate the Fourier expansions, which in turn can be employed to compute the values of $\tilde{\Psi}_{1}$ and $\tilde{\Psi}_{2}$ at CM points. 
A pleasant example is the CM point $z_{0}=\left(\zeta_{5}, \zeta_{5}^{2}\right)$ (where $\zeta_{5}=e^{2 \pi i / 5}$ ) corresponding to the cyclic CM extension $K=\mathbb{Q}\left(\zeta_{5}\right)$ of $F$. It is known that $z_{0}$ is an elliptic fixed point of $\Gamma$ of order 5 . The stabilizer of $z_{0}$ in $\Gamma$ is the cyclic subgroup generated by $\left(\begin{array}{cc}\omega^{\prime} & 1 \\ -1 & 0\end{array}\right)$. This implies that every Hilbert modular form for $\Gamma$ of weight coprime to 5 vanishes at $z_{0}$. In particular $g_{2}\left(z_{0}\right)=s_{6}\left(z_{0}\right)=0$. Consequently, only the term involving $s_{10}^{3}$ contributes to the value at $z_{0}$. We find that

$$
\begin{aligned}
\tilde{\Psi}_{1}\left(z_{0}\right) & =\Psi_{1}^{+}\left(z_{0}\right) / \Psi_{1}^{-}\left(z_{0}\right) \\
& =\frac{24527175191718750000+10968886216250000000 \sqrt{5}}{24527175191718750000-10968886216250000000 \sqrt{5}} \\
& =\frac{156973921227+70200871784 \sqrt{5}}{156973921227-70200871784 \sqrt{5}} \\
& =\left(\frac{\omega}{\omega^{\prime}}\right)^{27} \cdot \frac{\left(4+\omega^{\prime}\right)^{5} \cdot\left(5+\omega^{\prime}\right)^{5}}{(4+\omega)^{5} \cdot(5+\omega)^{5}} .
\end{aligned}
$$

Moreover,

$$
\begin{aligned}
\tilde{\Psi}_{2}\left(z_{0}\right) & =\Psi_{2}^{+}\left(z_{0}\right) / \Psi_{2}^{-}\left(z_{0}\right) \\
& =-\frac{68476004313518731312+30623400094519340937 \sqrt{5}}{68476004313518731312-30623400094519340937 \sqrt{5}} \\
& =\left(\frac{\omega}{\omega^{\prime}}\right)^{51} \cdot \frac{\left(9+\omega^{\prime}\right)^{5} \cdot\left(10+\omega^{\prime}\right)^{5}}{(9+\omega)^{5} \cdot(10+\omega)^{5}} .
\end{aligned}
$$

Notice that $4+\omega, 5+\omega, 9+\omega, 10+\omega$ are prime elements of $\mathcal{O}_{F}$ above 19, 29, 89, 109, respectively. In particular the same phenomenon as in $[\mathrm{BY}$ ] happens: the prime factors of the $\mathrm{CM}$ values are small.

We would like to evaluate $\tilde{\Psi}_{m}$ at other CM cycles as well, say, corresponding to CM extensions $K / F$ such that $K / \mathbb{Q}$ is non-Galois (and satisfies the assumptions of Section [6). Here it is rather difficult to compute the value exactly. To get a feeling for the problem, one can try to do some numerical computations. However, this is not so easy either, since the CM values will be large (or small) algebraic numbers in $F$. So from the floating point evaluation one cannot get the exact value. However, using the result of [BY] one obtains a convincing heuristic how the values should look like. With this extra information, the problem becomes accessible. For simplicity let us assume that $m$ is square-free and that $p=5$ as before. (For $p=13,17$ the same argument applies.)

The value of the Petersson metric of $H_{m}$ at $\mathcal{C M}(K)$ can be computed by means of the formula of $[\mathrm{BY}]$. Let $\mathfrak{l} \subset \mathcal{O}_{F}$ be a prime ideal above a prime $l \in \mathbb{Z}$. Using the notation of [BY], to $m$ and $l$ we can associate the quantity

$$
h_{m}(l)=\frac{W_{\tilde{K}}}{4}\left(b_{m p^{2}}(l)-b_{m}(l)\right) .
$$

As explained in [BY] (1.10) it should have a geometric interpretation as the intersection number of suitable models of $\operatorname{div}\left(H_{m}\right)$ and $\mathcal{C M}(K)$ in the fiber above $l$ of the moduli space 
of abelian surfaces with $\mathcal{O}_{F}$-action and $\partial_{F}^{-1}$-polarization. In the same way, over $\mathcal{O}_{F}$, we could associate to $m$ and $\mathfrak{l}$ the intersection number $h_{m}(\mathfrak{l})$ in the fiber above $\mathfrak{l}$. We should have

$$
h_{m}(l)= \begin{cases}2 h_{m}(\mathfrak{l}), & \text { if } l \text { is ramified in } \mathcal{O}_{F}, \\ h_{m}(\mathfrak{l}), & \text { if } l \text { is inert in } \mathcal{O}_{F}, \\ h_{m}(\mathfrak{l})+h_{m}\left(\mathfrak{l}^{\prime}\right), & \text { if } l \text { is split in } \mathcal{O}_{F} .\end{cases}
$$

According to (17.3) , one should be able to write $h_{m}(\mathfrak{l})=h_{m}^{+}(\mathfrak{l})+h_{m}^{-}(\mathfrak{l})$, where $h_{m}^{ \pm}(\mathfrak{l})$ denotes the intersection of $\operatorname{div}\left(\Psi_{m}^{ \pm}\right)$and $\mathcal{C} \mathcal{M}(K)$ in the fiber above $\mathfrak{l}$. Since $\left(\Psi_{m}^{+}\right)^{\prime}=\Psi_{m}^{-}$, we would have

$$
h_{m}^{+}(\mathfrak{l})=h_{m}^{-}\left(\mathfrak{l}^{\prime}\right) .
$$

In view of (17.2) the intersection number of $\operatorname{div}\left(\tilde{\Psi}_{m}\right)$ and $\mathcal{C} \mathcal{M}(K)$ in the fiber above $\mathfrak{l}$, would be given by

$$
h_{m}^{+}(\mathfrak{l})-h_{m}^{-}(\mathfrak{l})=h_{m}^{+}(\mathfrak{l})-h_{m}^{+}\left(\mathfrak{l}^{\prime}\right) .
$$

But since $\tilde{\Psi}_{m}$ has weight 0 , this quantity would be equal to $\operatorname{ord}_{\mathfrak{l}}\left(\tilde{\Psi}_{m}(\mathcal{C M}(K))\right)$. Consequently,

$$
\left|\operatorname{ord}_{\mathfrak{l}}\left(\tilde{\Psi}_{m}(\mathcal{C} \mathcal{M}(K))\right)\right| \leq \begin{cases}0, & \text { if } l \text { is ramified or inert in } \mathcal{O}_{F}, \\ h_{m}(l), & \text { if } l \text { is split in } \mathcal{O}_{F} .\end{cases}
$$

The quantities $h_{m}(l)$ can be computed by means of the formula of [BY]. In that way there are only finitely many possibilities left for the prime ideal factorization of $\tilde{\Psi}_{m}(\mathcal{C} \mathcal{M}(K))$. Using a computer algebra system one can now compute the $\mathrm{CM}$ value if the Fourier expansion of $\tilde{\Psi}_{m}$ is known.

In the special cases $p=5$ and $m=1,2$ considered before, we computed a few CM values corresponding to non-biquadratic CM fields $K$. We listed some data on a few CM fields in Table 3, including the class number $h_{K}$, and a system of representatives for the ideal class group of $K$. The corresponding CM values are given in Tables 1 and 2

Combining the above considerations with Corollary 1.3 of [BY] we are lead to the following conjecture.

Conjecture 7.1. Let $m$ be a positive integer, and let $\mathfrak{l} \subset \mathcal{O}_{F}$ be a prime ideal above a prime $l \in \mathbb{Z}$ such that $\operatorname{ord}_{\mathfrak{l}}\left(\tilde{\Psi}_{m}(\mathcal{C} \mathcal{M}(K))\right) \neq 0$. Then $\mathfrak{l}^{\prime} \neq \mathfrak{l}$ and $4 l \mid m^{2} p^{2} q-r^{2}$ for some $r \in \mathbb{Z}$ with $|r|<m p \sqrt{q}$.

\section{FURTHER REMARKS AND OPEN PROBLEMS}

1. Is there a nice moduli interpretation of $\tilde{\Psi}_{1}$ as an invariant of abelian surfaces with $\mathcal{O}_{F}$-multiplication and $\partial_{F}^{-1}$-polarization?

2. In the numerical calculations it always happened that

$$
\tilde{\Psi}_{m}\left(\mathcal{C} \mathcal{M}\left(K, \Phi, \mathcal{O}_{F}\right)\right)=\tilde{\Psi}_{m}\left(\mathcal{C} \mathcal{M}\left(K, \sigma^{3} \Phi, \mathcal{O}_{F}\right)\right) .
$$

This explains that the CM values in Tables 1 and 2 are squares. Is it possible to prove this in general? (See also Remark 6.4.) Moreover, it is striking that the CM values 
TABLE 1. CM values of $\tilde{\Psi}_{1}$ for $\mathbb{Q}(\sqrt{5})$

\begin{tabular}{|r|l|l|}
\hline$q$ & $\prod_{l} l^{h_{1}(l)}$ & $\tilde{\Psi}_{1}(\mathcal{C} \mathcal{M}(K))$ \\
\hline 5 & $5^{40} \cdot 19^{10} \cdot 29^{10}$ & $\left(\frac{\omega}{\omega^{\prime}}\right)^{54} \frac{\left(4+\omega^{\prime}\right)^{10} \cdot\left(5+\omega^{\prime}\right)^{10}}{(4+\omega)^{10} \cdot(5+\omega)^{10}}$ \\
\hline 41 & $2^{16} \cdot 5^{42} \cdot 23^{4} \cdot 31^{4} \cdot 37^{2} \cdot 61^{4} \cdot 107^{2} \cdot 127^{2}$ & $\left(\frac{\omega}{\omega^{\prime}}\right)^{32} \frac{(5+2 \omega)^{2} \cdot\left(7+3 \omega^{\prime}\right)^{2}}{\left(5+2 \omega^{\prime}\right)^{2} \cdot(7+3 \omega)^{2}}$ \\
\hline 61 & $3^{12} \cdot 5^{42} \cdot 13^{4} \cdot 41^{4} \cdot 83^{2} \cdot 103^{2} \cdot 109^{2} \cdot 113^{2} \cdot 199^{2} \cdot 379^{2}$ & $\left(\frac{\omega}{\omega^{\prime}}\right)^{24} \frac{(6+\omega)^{2} \cdot\left(10+\omega^{\prime}\right)^{2} \cdot(13+3 \omega)^{2} \cdot\left(19+\omega^{\prime}\right)^{2}}{\left(6+\omega^{\prime}\right)^{2} \cdot(10+\omega)^{2} \cdot\left(13+3 \omega^{\prime}\right)^{2} \cdot(19+\omega)^{2}}$ \\
\hline 109 & $3^{12} \cdot 5^{40} \cdot 7^{6} \cdot 43^{2} \cdot 61^{4} \cdot 71^{4} \cdot 73^{2} \cdot 97^{2} \cdot 113^{2} \cdot 223^{2} \cdot 409^{2} \cdot 499^{2}$ & $\left(\frac{\omega}{\omega^{\prime}}\right)^{12} \frac{(7+3 \omega)^{2} \cdot(8+\omega)^{2} \cdot(19+3 \omega)^{2} \cdot(20+9 \omega)^{2}}{\left(7+3 \omega^{\prime}\right)^{2} \cdot\left(8+\omega^{\prime}\right)^{2} \cdot\left(19+3 \omega^{\prime}\right)^{2} \cdot\left(20+9 \omega^{\prime}\right)^{2}}$ \\
\hline 241 & $2^{50} \cdot 3^{36} \cdot 5^{126} \cdot 29^{14} \cdot 47^{6} \cdot 53^{6} \cdot 61^{8} \cdot 67^{2} \cdot 83^{2} \cdot 97^{6} \cdot 229^{4} \cdot$ & $\left(\frac{\omega}{\omega^{\prime}}\right)^{84} \frac{\left(5+\omega^{\prime}\right)^{2} \cdot\left(17+3 \omega^{\prime}\right)^{2}}{(5+\omega)^{2} \cdot(17+3 \omega)^{2}}$ \\
\hline 281 & $2^{44} \cdot 5^{128} \cdot 7^{22} \cdot 17^{12} \cdot 43^{6} \cdot 53^{6} \cdot 59^{6} \cdot 101^{4} \cdot 109^{6} \cdot 137^{2} \cdot 191^{4} \cdot$ & $\left(\frac{\omega}{\omega^{\prime}}\right)^{72} \frac{(7+2 \omega)^{2} \cdot(10+\omega)^{2} \cdot(13+2 \omega)^{2} \cdot(19+4 \omega)^{2}}{\left(7+2 \omega^{\prime}\right)^{2} \cdot\left(10+\omega^{\prime}\right)^{2} \cdot\left(13+2 \omega^{\prime}\right)^{2} \cdot\left(19+4 \omega^{\prime}\right)^{2}}$ \\
\hline 409 & $317^{2} \cdot 421^{4} \cdot 647^{2} \cdot 787^{2} \cdot 857^{2} \cdot 877^{2}$ & $2^{48} \cdot 3^{42} \cdot 5^{120} \cdot 17^{12} \cdot 23^{14} \cdot 53^{10} \cdot 83^{4} \cdot 103^{6} \cdot 109^{6} \cdot 167^{2} \cdot$ \\
& $179^{4} \cdot 197^{2} \cdot 239^{4} \cdot 349^{4} \cdot 571^{4} \cdot 1187^{2} \cdot 1277^{2}$ & $\left(\frac{\omega}{\omega^{\prime}}\right)^{72} \frac{(10+\omega)^{2} \cdot(23+2 \omega)^{2}}{\left(10+\omega^{\prime}\right)^{2} \cdot\left(23+2 \omega^{\prime}\right)^{2}}$ \\
\hline
\end{tabular}

TABLE 2. CM values of $\tilde{\Psi}_{2}$ for $\mathbb{Q}(\sqrt{5})$

\begin{tabular}{|r|l|l|}
\hline$q$ & $\prod_{l} l^{h_{2}(l)}$ & $\tilde{\Psi}_{2}(\mathcal{C M}(K))$ \\
\hline 5 & $2^{120} \cdot 5^{50} \cdot 89^{10} \cdot 109^{10}$ & $\left(\frac{\omega}{\omega^{\prime}}\right)^{102} \frac{\left(9+\omega^{\prime}\right)^{10} \cdot\left(10+\omega^{\prime}\right)^{10}}{(9+\omega)^{10} \cdot(10+\omega)^{10}}$ \\
\hline 41 & $2^{104} \cdot 5^{48} \cdot 23^{4} \cdot 37^{2} \cdot 41^{2} \cdot 43^{2} \cdot 73^{2} \cdot 83^{2} \cdot$ & $\left(\frac{\omega}{\omega^{\prime}}\right)^{84} \frac{(6+\omega)^{2} \cdot(17+5 \omega)^{2} \cdot(19+8 \omega)^{2} \cdot\left(25+9 \omega^{\prime}\right)^{2} \cdot\left(26+9 \omega^{\prime}\right)^{2} \cdot(29+8 \omega)^{2}}{\left(6+\omega^{\prime}\right)^{2} \cdot\left(17+5 \omega^{\prime}\right)^{2} \cdot\left(19+8 \omega^{\prime}\right)^{2} \cdot\left(25+9 \omega^{2} \cdot(26+9 \omega)^{2} \cdot\left(29+8 \omega^{\prime}\right)^{2}\right.}$ \\
& $113^{2} \cdot 349^{2} \cdot 449^{2} \cdot 769^{2} \cdot 829^{2} \cdot 1009^{2}$ & \\
\hline 61 & $2^{120} \cdot 3^{14} \cdot 55^{4} \cdot 13^{2} \cdot 41^{4} \cdot 47^{4} \cdot 61^{2} \cdot 73^{2} \cdot$ & $\left(\frac{\omega}{\omega^{\prime}}\right)^{80} \frac{\left(6+\omega^{\prime}\right)^{2} \cdot(7+3 \omega)^{2} \cdot\left(14+3 \omega^{\prime}\right)^{2} \cdot(35+11 \omega)^{2}}{(6+\omega)^{2} \cdot\left(7+3 \omega^{\prime}\right)^{2} \cdot(14+3 \omega)^{2} \cdot\left(35+11 \omega^{\prime}\right)^{2}}$ \\
& $229^{2} \cdot 283^{2} \cdot 443^{2} \cdot 503^{2} \cdot 1489^{2}$ & $\left(\frac{\omega}{\omega^{\prime}}\right)^{66} \frac{(15+7 \omega)^{2} \cdot\left(34+13 \omega^{\prime}\right)^{2} \cdot(47+15 \omega)^{2}}{\left(15+7 \omega^{\prime}\right)^{2} \cdot(34+13 \omega)^{2} \cdot\left(47+15 \omega^{\prime}\right)^{2}}$ \\
\hline 109 & $2^{120} \cdot 3^{12} \cdot 5^{50} \cdot 7^{12} \cdot 43^{2} \cdot 263^{2} \cdot 281^{4} \cdot 307^{2} \cdot$ \\
& $523^{2} \cdot 683^{2} \cdot 823^{2} \cdot 1429^{2} \cdot 2689^{2}$ &
\end{tabular}

$\tilde{\Psi}_{1}\left(\mathcal{C} \mathcal{M}\left(K, \Phi, \mathcal{O}_{F}\right)\right)$ corresponding to non-Galois CM fields are actually square-free elements of $F$. Is this a coincidence or a general phenomenon? Notice that by Corollary 6.3 this would imply that $\tilde{\Psi}_{1}(z)$ generates the class field $L_{K}$. This is the case with the examples computed above.

3. Is there a (finite) subset $B$ of the positive integers such that the functions $\tilde{\Psi}_{m}(m \in B)$ generate the function field of the symmetric Hilbert modular surface corresponding to $\mathrm{SL}_{2}\left(\mathcal{O}_{F}\right)$ ?

4. In terms of the generators $g_{2}, s_{6}, s_{10}$ of the ring of symmetric Hilbert modular forms of even weight for $\mathbb{Q}(\sqrt{5})$ the functions $\tilde{\Psi}_{1}$ and $\tilde{\Psi}_{2}$ look rather complicated. Are there other generators which yield a nicer description (possibly of all $\tilde{\Psi}_{m}$ )? 
TABLE 3. CM extensions of $\mathbb{Q}(\sqrt{5})$

\begin{tabular}{|r|l|l|l|}
\hline$q$ & $K=F(\sqrt{\Delta})$ & $h_{K}$ & $\mathrm{CL}(K)$ \\
\hline 5 & $\Delta=-\frac{5+\sqrt{5}}{2}$ & 1 & $\mathcal{O}_{K}=\mathcal{O}_{F}+\sqrt{\Delta} \mathcal{O}_{F}$ \\
\hline 41 & $\Delta=-\frac{13+\sqrt{5}}{2}$ & 1 & $\mathcal{O}_{K}=\mathcal{O}_{F}+\frac{1}{2}\left(\sqrt{\Delta}+\frac{3+\sqrt{5}}{2}\right) \mathcal{O}_{F}$ \\
\hline 61 & $\Delta=-(9+2 \sqrt{5})$ & 1 & $\mathcal{O}_{K}=\mathcal{O}_{F}+\frac{1}{2}(\sqrt{\Delta}+1) \mathcal{O}_{F}$ \\
\hline 109 & $\Delta=-\frac{21+\sqrt{5}}{2}$ & 1 & $\mathcal{O}_{K}=\mathcal{O}_{F}+\frac{1}{2}\left(\sqrt{\Delta}+\frac{3+\sqrt{5}}{2}\right) \mathcal{O}_{F}$ \\
\hline 241 & $\Delta=-\frac{33+5 \sqrt{5}}{2}$ & 3 & $\begin{array}{l}\mathcal{O}_{K}=\mathcal{O}_{F}+\frac{1}{2}\left(\sqrt{\Delta}+\frac{3+\sqrt{5}}{2}\right) \mathcal{O}_{F}, \\
\mathfrak{A}=2 \mathcal{O}_{F}+\frac{1}{2}\left(\sqrt{\Delta}+\frac{9+3 \sqrt{5}}{2}\right) \mathcal{O}_{F}, \\
\mathfrak{B}=4 \mathcal{O}_{F}+\frac{1}{2}\left(\sqrt{\Delta}+\frac{9+3 \sqrt{5}}{2}\right) \mathcal{O}_{F}\end{array}$ \\
\hline 281 & $\Delta=-\frac{37+7 \sqrt{5}}{2}$ & 3 & $\begin{array}{l}\mathcal{O}_{K}=\mathcal{O}_{F}+\frac{1}{2}\left(\sqrt{\Delta}+\frac{1+\sqrt{5}}{2}\right) \mathcal{O}_{F}, \\
\mathfrak{A}=2 \mathcal{O}_{F}+\frac{1}{2}\left(\sqrt{\Delta}+\frac{1+\sqrt{5}}{2}\right) \mathcal{O}_{F}, \\
\mathfrak{B}=4 \mathcal{O}_{F}+\frac{1}{2}\left(\sqrt{\Delta}+\frac{9+\sqrt{5}}{2}\right) \mathcal{O}_{F}\end{array}$ \\
\hline 409 & $\Delta=-\frac{41+3 \sqrt{5}}{2}$ & 3 & $\begin{array}{l}\mathcal{O}_{K}=\mathcal{O}_{F}+\frac{1}{2}\left(\sqrt{\Delta}+\frac{1+\sqrt{5}}{2}\right) \mathcal{O}_{F}, \\
\mathfrak{A}=2 \mathcal{O}_{F}+\frac{1}{2}\left(\sqrt{\Delta}+\frac{7+3 \sqrt{5}}{2}\right) \mathcal{O}_{F}, \\
\mathfrak{B}=4 \mathcal{O}_{F}+\frac{1}{2}\left(\sqrt{\Delta}+\frac{-1+3 \sqrt{5}}{2}\right) \mathcal{O}_{F}\end{array}$ \\
\hline
\end{tabular}

5. Describe how the correspondence of Theorem 5.2 behaves under the action of the corresponding Hecke algebras. It should be Hecke equivariant, where the Hecke action on the image is multiplicative (see also [Bo2] Problem 16.5).

6. Generalize the results of the present paper to Hilbert modular surfaces of arbitrary discriminant.

\section{REFERENCES}

[AbSt] M. Abramowitz and I. Stegun, Pocketbook of Mathematical Functions, Verlag Harri Deutsch, Thun (1984).

[Ap] T. M. Apostol, Introduction to Analytic Number Theory, Springer-Verlag (1976).

[Bo1] R. E. Borcherds, Automorphic forms on $\mathrm{O}_{s+2,2}(\mathbb{R})$ and infinite products, Invent. Math. 120 (1995),161-213.

[Bo2] R. E. Borcherds, Automorphic forms with singularities on Grassmannians, Invent. Math. 132 (1998), 491-562.

[Br1] J. H. Bruinier, Borcherds products and Chern classes of Hirzebruch-Zagier divisors, Invent. math. 138 (1999), 51-83.

[Br2] J. H. Bruinier, Borcherds products on $\mathrm{O}(2, l)$ and Chern classes of Heegner divisors, Lect. Notes Math. 1780, Springer-Verlag, Berlin (2002). 
[BB] J. H. Bruinier and M. Bundschuh, On Borcherds products associated with lattices of prime discriminant, Ramanujan J. 7 (2003), 49-61.

[BY] J. H. Bruinier and T. Yang, CM values of Hilbert modular functions, preprint (2004).

$[\mathrm{CH}] \quad$ P. E. Conner and J. Hurrelbrink, Class Number Parity, Series in Pure Math. vol. 8, World Scientific (1988).

[DN] K. Doi and H. Naganuma, On the functional equation of certain Dirichlet series, Invent. Math. 9 (1969), 1-14.

[Ge] G. van der Geer, Hilbert Modular Surfaces, Springer-Verlag, Berlin (1988).

[Go] E. Z. Goren, Lectures on Hilbert Modular Varieties and Modular Forms, CRM Monograph Series 14, American Mathematical Society (2002).

[GZ] B. Gross and D. Zagier, On singular moduli, J. Reine Angew. Math. 355 (1985), 191-220.

[Gu] K.-B. Gundlach, Die Bestimmung der Funktionen zur Hilbertschen Modulgruppe des Zahlkörpers $\mathbb{Q}(\sqrt{5})$, Math. Annalen 152 (1963), 226-256.

[He] D. A. Hejhal, The Selberg Trace Formula for PSL $(2, \mathbb{R})$, Lecture Notes in Mathematics 1001, Springer-Verlag (1983).

[HZ] F. Hirzebruch and D. Zagier, Intersection Numbers of Curves on Hilbert Modular Surfaces and Modular Forms of Nebentypus, Invent. Math. 36 (1976), 57-113.

[La] S. Lang, Algebraic Number Theory, Addison-Wesley, 1970.

[Mü] R. Müller, Hilbertsche Modulfunktionen zu $\mathbb{Q}(\sqrt{5})$, Arch. Math. 45, 239-251 (1985).

[Na] H. Naganuma, On the coincidence of two Dirichlet series associated with cusp forms of Hecke's "Neben"-type and Hilbert modular forms over a real quadratic field, J. Math. Soc. Japan 25 (1973), 547-555.

[Ni] D. Niebur, A class of nonanalytic automorphic functions, Nagoya Math. J. 52 (1973), 133-145.

[Ro] J. Rouse, A Relation Between the Fourier Coefficients and the Values of Holomorphic Modular Functions, in preparation.

[Sh] G. Shimura, Abelian varieties with complex multiplication and modular functions, Princeton Univ. Press (1998).

[Za1] D. Zagier, Modular Forms Associated to Real Quadratic Fields, Invent. Math. 30 (1975), 1-46.

[Za2] D. Zagier, Modular forms whose Fourier coefficients involve zeta-functions of quadratic fields. In: Modular Functions of One Variable VI, Lecture Notes in Math. 627, Springer-Verlag (1977), $105-169$.

[Za3] D. Zagier, Traces of singular moduli, Motives, polylogarithms, and Hodge theory (Eds. F. Bogomolov and L. Katzarkov) I, Intl. Press, Somerville (2003), 211-244.

[Zh] S.W. Zhang, Equidistribution of CM-points on quaternion Shimura varieties, preprint (2004), pp35.

Mathematisches Institut, Universität Zu Köln, Weyertal 86-90, D-50931 Köln, Germany

E-mail address: bruinier@math.uni-koeln.de

Department of Mathematics, University of Wisconsin Madison, Van Vleck Hall, MadiSON, WI 53706, USA

E-mail address: thyang@math.wisc.edu 